\title{
A Two-Fluid Conditional Averaging Paradigm for the Theory and Modeling of Turbulent Premixed Combustion
}

\author{
Vladimir L. Zimont \\ CRS4 Research Center POLARIS, 09010 Pula (CA), Italy \\ Correspondence should be addressed to Vladimir L. Zimont; zimont@crs4.it
}

Received 20 November 2018; Accepted 3 June 2019; Published 7 August 2019

Academic Editor: Bruce Chehroudi

Copyright ( 2019 Vladimir L. Zimont. This is an open access article distributed under the Creative Commons Attribution License, which permits unrestricted use, distribution, and reproduction in any medium, provided the original work is properly cited.

\begin{abstract}
This paper extends a recent theoretical study that was previously presented in the form of a brief communication (Zimont, C\&F, 192, 2018, 221-223), in which we proposed a simple splitting method for the derivation of two-fluid conditionally averaged equations of turbulent premixed combustion in the flamelet regime, formulated more conveniently for applications involving unclosed equations without surface-averaged unknowns. This two-fluid conditional averaging paradigm avoids the challenge in the Favre averaging paradigm of modeling the countergradient scalar transport phenomenon and the unusually large velocity fluctuations in a turbulent premixed flame. It is a more suitable conceptual framework that is likely to be more convenient in the long run than the traditional Favre averaging method. In this article, we further develop this paradigm and pay particular attention to the problem of modeling turbulent premixed combustion in the context of a two-fluid approach. We formulate and analyze the unclosed differential equations in terms of the conditions of the Reynolds stresses $\tau_{i j, u}, \tau_{i j, b}$ and the mean chemical source $\overline{\rho W}$, which are the only modeling unknowns required in our alternative conditionally averaged equations. These equations are necessary for the development of model differential equations for the Reynolds stresses and the chemical source in the advanced modeling and simulation of turbulent premixed combustion. We propose a simpler approach to modeling the conditional Reynolds stresses based on the use of the twofluid conditional equations of the standard " $k-\varepsilon$ " turbulence model, which we formulate using the splitting method. The main problem arising here is the appearance in these equations of unknown terms describing the exchange of the turbulent energy $k$ and dissipation rate $\varepsilon$ in the unburned and burned gases. We propose an approximate way to avoid this problem. We formulate a simple algebraic expression for the mean chemical source that follows from our previous theoretical analysis of the transient turbulent premixed flame in the intermediate asymptotic stage, in which small-scale wrinkles in the instantaneous flame surface reach statistical equilibrium, while the large-scale wrinkles remain in statistical nonequilibrium.
\end{abstract}

\section{Introduction}

We use the term 'paradigm' in the title of this article to emphasize that the two-fluid approach is a conceptual framework for analyzing and modeling turbulent premixed combustion in the flamelet regime. Paradigms in turbulent combustion research, with their basic conceptual viewpoints and principles of theory and modeling, have been discussed in the literature (see, for example, the paper entitled "Paradigm in Turbulent Combustion Research" by Bilger et al. [1], which contains relevant citations). In this paper, we primarily analyze turbulent premixed combustion in the context of "Damköhler's paradigm" [1], which implies that instantaneous combustion takes place in a strongly wrinkled laminar flame, and we touch only briefly upon the thickened flamelet regime. Bray, Moss, and Libby proposed the progress variable $c$ for use in the description of premixed combustion of a single quantity and developed BML formalism, which forms the basis of the BML model developed using the Favre ensemble averaging method [2]. This more elaborate Damköhler's laminar flamelet paradigm can be referred to as the "BML Favre averaging paradigm". We remind readers that this paradigm leads to the emergence of a fundamental difficulty in the theory and modeling of turbulent premixed combustion involving the adequate prediction of the unknown mean flux of the progress variable $\overrightarrow{\rho \vec{u}^{\prime \prime} c^{\prime \prime}}$ and stress tensor $\overrightarrow{\rho \vec{u}^{\prime \prime} \vec{u}^{\prime \prime}}$, which appear in the unclosed 
Favre-averaged combustion and hydrodynamics equations of the problem. The point is that the scalar flux $\overrightarrow{\rho \vec{u}^{\prime \prime} c^{\prime \prime}}$ is predominantly (but not always) countergradient (sometimes called 'countergradient turbulent diffusion'), and the stress tensor $\overrightarrow{\rho \vec{u}} \vec{u}^{\prime \prime}$ strongly depends on abnormally large velocity fluctuations observed in the turbulent premixed flame. The gas dynamic (nonturbulent) nature of these phenomena was clearly explained by Pope in [3]:

"As well as looking at the detail structure of a turbulent premixed flame, we can examine mean quantities. Here too, in comparison to other turbulent flows, there are some unusual observations, the most striking being countergradient diffusion. Within the flame there is a mean flux of reactants due to the fluctuating component of the velocity field. Contrary to normal expectations and observations in other flows, it is found that this flux transports reactants up the mean-reactants gradient, away from the products (hence countergradient diffusion). A second notable phenomenon is the large production of turbulent energy within the flame: Behind the flame the velocity variance can be 20 times its upstream value. Both these phenomena result from the large density difference between reactants and products and from the pressure field due to volume expansion [...] From the Euler equations it is readily seen that a given pressure gradient accelerates the light products more than the heavier reactants. This mechanism is responsible both for countergradient diffusion and for turbulent energy production".

Many articles have been devoted to theoretical and experimental studies of these phenomena. More advanced theoretical and modeling approaches in the context of the BML Favre averaging paradigm use the Favre-averaged unclosed equations in terms of the components of the scalar flux $\overline{\rho u_{i}^{\prime \prime} c^{\prime \prime}}$ and stress tensor $\overline{\rho u_{i}^{\prime \prime} u_{j}^{\prime \prime}}$, and further approximation of the unknowns appears within turbulence theories (see, for example, [4]). To justify the need for our study, we emphasize that the countergradient scalar flux and abnormally large velocity fluctuations cannot be described adequately in the context of the BML Favre averaging paradigm (as we will illustrate below using as an example the results obtained in [4]). The reason for this is that these phenomena are caused by the large difference in the conditional mean velocities $\overline{\vec{u}}_{u}$ and $\overline{\vec{u}}_{b}$, due to the different pressure-driven acceleration of the heavier unburned and lighter burned gases. These velocities, which cannot be predicted by the Favre-averaged equations, are at the same time described directly by the twofluid conditional equations. It would be appropriate here to quote a statement made by Forman Williams in his Hottel lecture entitled "The Role of Theory in Combustion Science" [5]:

"It is relevant to distinguish between the science and the technology of the subject. The march of technology has never hesitated. It uses science whenever possible but often, especially in combustion, forges ahead by trial and error, or fortuitously by application of scientific misconception, but without scientific understanding".

In this context, the use of Favre averaging in the modeling of turbulent premixed combustion in the flamelet regime is, strictly speaking, the "application of scientific misconception", in contrast to two-fluid conditional averaging, which is conceptually adequate. Hence, two fundamental modeling problems arise in the turbulent combustion theory developed in the framework of the Favre averaging paradigm: the issue of adequate prediction in the premixed flame of both phenomena ("countergradient turbulent diffusion" and "unusually large generation of turbulence") disappears in the framework of the two-fluid conditional average paradigm.

As far as we know, the pioneers of the theoretical two-fluid approach to turbulent premixed combustion were Spalding [6] and Weller [7]. In [6], Spalding also analyzed some previous papers related to the two-fluid approach. In [7], Weller presented conditionally averaged two-fluid equations that in fact were model equations. The exact, unclosed, two-fluid conditionally averaged mass and momentum equations, which served as a starting point for our study, were obtained in [8]. This paper extends our recent theoretical development of the two-fluid theory of turbulent premixed combustion described in [9] in the format of a brief communication, in which we proposed a simple and physically clear splitting method for the derivation of the unclosed two-fluid conditional equations of turbulent premixed combustion. This method made it possible to rederive the conditional equations obtained in [8] and to understand the reason for the appearance of surface-averaged variables in these, the impossibility of the well-founded modeling of which is one of the obstacles to using this approach in applications; in addition, this allowed us to indicate a way of excluding their appearance. The only required modeling unknowns appearing in the original alternative two-fluid conditional equations formulated in [9] are the conditional Reynolds stress tensors $\tau_{u}=-\rho_{u}\left(\overrightarrow{\vec{u}}^{\prime} \vec{u}^{\prime}\right)_{u}$ and $\tau_{b}=-\rho_{u}\left(\overrightarrow{\vec{u}}^{\prime} \vec{u}^{\prime}\right)_{b}$ in the unburned and burned gases, and the mean chemical source $\overline{\rho W}$.

This paper makes the following contributions:

(i) We describe in more detail the splitting method and the derivation of the unclosed two-fluid conditionally averaged equations, both those described in the literature and the alternative versions.

(ii) We obtain the unclosed equations in terms of the conditional moments $\left(\overline{u_{i}^{\prime} u_{j}^{\prime}}\right)_{u}$ and $\left(\overline{u_{i}^{\prime} u_{j}^{\prime}}\right)_{b}$, by splitting the known unclosed equations in terms of the Favre average $\left(\overline{\rho u_{i}^{\prime \prime} u_{j}^{\prime \prime}}\right)$, and present the unclosed equation for the mean chemical source $\overline{\rho W}$, which can be used for advanced modeling of the unknowns in the twofluid conditionally averaged equations.

(iii) We formulate simple models for estimating the unknown chemical source $\overline{\rho W}$ in the laminar and 
thickened flamelet combustion regimes, based on our previous theoretical studies of transient turbulent premixed flames in the intermediate asymptotic stage, which were published elsewhere.

(iv) We reformulate the equations for the classical " $k-\varepsilon$ " turbulence model in terms of the conditional turbulent energy and dissipation rate using the slipping method and estimate the potentialities and problems arising from the use of the obtained two-fluid " $k$ $\varepsilon$ " model to model turbulent characteristics and the conditional Reynolds stresses $\tau_{u}$ and $\tau_{b}$.

We consider the two-fluid approach to the theory and modeling of turbulent premixed combustion in the flamelet regime as a promising alternative in the long run to traditional methods based on Favre ensemble averaging. The two-fluid conditionally averaged equations adequately describe the hydrodynamic (due to the different pressure-driven acceleration of the unburned and burned gases) and turbulent (using the corresponding two-fluid unclosed equation and corresponding two-fluid turbulence model) effects and their interaction in the turbulent premixed flame. This allows us to eliminate the necessity of modeling the mean scalar flux and stress tensor, which presents a challenge in the context of the Favre averaging framework.

A strong argument in favor of the two-fluid approach is provided by a review [10] of many studies of the structure of the instantaneous combustion zone in turbulent mixed flames, which showed that combustion occurs in thin, strongly wrinkled flamelet sheets: "Thin flamelets are found to occur even when the Karlovitz number greatly exceeds unity. The preheat zone average thickness is no larger than the laminar value in many studies, while in some cases it is 2-4 times larger." The two-fluid approach is applicable for modeling not only premixed combustion in the laminar flamelet regime, but also flames in the thickened flamelet regime. We draw the reader's attention to the paper [11] quoted in [10], where the local flame structure of a premixed swirl-stabilized gas turbine burner has been investigated. We consider the result obtained in [11] that the flamelet thermal thickness in the investigated highly turbulent lean premixed flames is close to the thermal thickness of the laminar flame as a significant argument in favor of the use of the two-fluid approach.

The two-fluid conditionally averaged unclosed equations directly describe the conditional mean velocities $\overrightarrow{\vec{u}}_{u}$ and $\overline{\vec{u}}_{b}$, the conditional mean pressures $\bar{p}_{u}$ and $\bar{p}_{b}$, the mean progress variable $\bar{c}$, and hence the probabilities of unburned gas $P_{u}=$ $1-\bar{c}$ and burned gas $P_{b}=\bar{c}$. These can be used to calculate the mean density $\bar{\rho}$ and pressure $\bar{p}$, the Favre-averaged progress variable $\tilde{c}=\overline{\rho c} / \bar{\rho}$, the Reynolds- and Favre-averaged velocities $\overline{\vec{u}}$ and $\widetilde{\vec{u}}$, and the mean scalar flux $\overline{\rho \vec{u}}{ }^{\prime \prime} c^{\prime \prime}$. In conjunction with the corresponding combustion and turbulence models, these equations also describe the mean chemical source $\overline{\rho W}$, the conditional Reynolds stresses $\tau_{i j}^{u}=-\rho_{u}\left(\overline{u_{i}^{\prime} u_{j}^{\prime}}\right)_{u}$ and $\tau_{i j}^{b}=$ $-\rho_{u}\left(\overline{u_{i}^{\prime} u_{j}^{\prime}}\right)_{b}$, the Favre-averaged stresses $\tau_{i j}=-\overline{\rho u_{i}^{\prime \prime} u_{j}^{\prime \prime}}$, and some other turbulent characteristics.
In our study, together with the conditional mean variables, we also use the Reynolds- and Favre-averaged ones, which are useful in the interpretation of the results of numerical simulations and comparison with experimental data. However, the chemical source $\overline{\rho W}$, which depends on curtain statistical characteristics of randomly wrinkled instantaneous flame, is not a conditional mean characteristic. The source term $\overline{\rho W}$ represents the production rate per unit volume of the product. The rate per unit mass is a Favreaveraged characteristic $\widetilde{W}=\overline{\rho W} / \bar{\rho}$. The unclosed equation for the chemical source, which is considered in this paper, was formulated in [12] in terms of $\widetilde{W}$. We express the variables $\bar{\rho}$ and $\widetilde{\vec{u}}$ appearing in this equation using $\bar{c}, \overline{\vec{u}}_{u}$, and $\overline{\vec{u}}_{b}$, which are directly described by the two-fluid conditional equations.

The paper is organized as follows:

Section 2: The two-fluid mathematical model and the conditions of its applicability to premixed combustion in the laminar and thickened flamelet regimes are described.

Section 3: The potentialities and limitations of the Favre and two-fluid conditional averaging frameworks are discussed, and the aim of the study is formulated.

Section 4: A splitting method for the derivation of unclosed two-fluid conditional equations is presented; it explains the origin of surface-averaged unknowns and demonstrates how to avoid them.

Section 5: Two statistical concepts of premixed combustion in the flamelet regime are described in the form of a three-stage and a global one-stage process, resulting in equations with and without surface-averaged unknowns, respectively.

Section 6: An alternative system is presented for the twofluid unclosed equations, in which the only unknowns that need to be modeled are the conditional Reynolds stresses and the chemical source.

Section 7: Unclosed equations are obtained for the conditional Reynolds stress using the splitting method.

Section 8: Unclosed equations for the mean chemical source are presented and analyzed.

Section 9: Equations for the conditional mean turbulent energy and dissipation rate are obtained by splitting the Favre-averaged equations of the classical " $k-\varepsilon$ " turbulence model.

Section 10: The chemical source is modeled for transient flames in the laminar and thickened regimes.

Section 11: A summary of this work and a conclusion are presented.

Appendix: The hydrodynamic/hydraulic analytical theory of the countergradient scalar flux is described in detail, and its applications for impinging and Bunsen flames and criteria for transition of the scalar flux are discussed.

The preliminary results of the current paper have been presented at three conferences (Zimont, V.L., Gas Dynamics in Turbulent Premixed Combustion: Conditionally Averaged Unclosed Equations and Analytical Formulation of the Problem, the $23^{\text {rd }}$ International Colloquium on the Dynamics of Explosions and Reactive System, University of California, Irvine, USA, July 24-29, 2011, pp. 1-6. Zimont, V.L., An Alternative Approach to Modeling Turbulent Premixed 


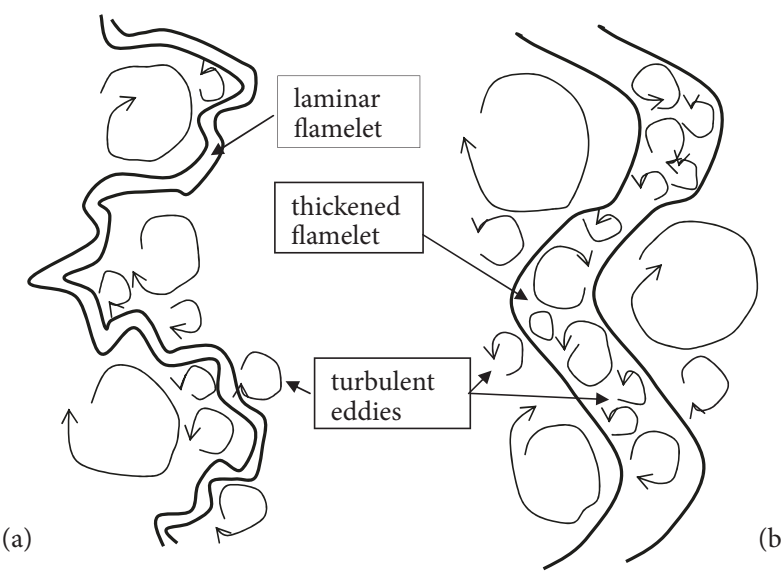

(b)

Figure 1: Laminar flamelet (a) and microturbulent (thickened) flamelet (b) combustion mechanisms.

Combustion, the Seventh Mediterranean Combustion Symposium, Chia Laguna, Cagliari, Sardinia, Italy, September 1115, 2011, pp. 1-11. Zimont, V.L., An Approach in Turbulent Premixed Combustion Research Based on Conditional Averaging, Joint Meeting of the British and Scandinavian-Nordic Section of the Combustion Institute, Cambridge, UK, March 27-28, 2014, pp. 67-68.).

\section{The Two-Fluid Mathematical Model and Its Applicability to Premixed Combustion}

In this section, we consider the conditions for applicability of the two-fluid mathematical model (in which it is postulated that instantaneous combustion takes place in a wrinkled flame of zero thickness) to combustion in the flamelet regime (where instantaneous combustion takes place in a strongly wrinkled laminar flame) and to combustion in the thickened (microturbulent) flamelet regime, where eddies smaller than the laminar flame thickness penetrate and thicken the preheat zone of the instantaneous flame (Figure 1).

2.1. The Two-Fluid Mathematical Model. The two-fluid mathematical model corresponds to the limiting case in which the width of the instantaneous laminar flame $\delta_{L}$ is zero; i.e., instantaneous combustion takes place in a strongly wrinkled flame surface that propagates in a direction normal to itself with speed $S_{L}$. Thus, the turbulent flame in the "flame surface regime" consists of two fluids: entirely unburned and completely burned gases.

This model corresponds to a limiting case of the laminar flame, in which the molecular transfer coefficient $\chi$ and chemical time $\tau_{c h}$ in the known expressions for the speed $S_{L} \approx\left(\chi / \tau_{c h}\right)^{1 / 2}$ and width $\delta_{L} \approx\left(\chi \cdot \tau_{c h}\right)^{1 / 2}$ tend to zero, $\chi \longrightarrow 0, \tau_{c h} \longrightarrow 0$, while their ratio tends to a constant $\left(\chi / \tau_{c h}\right) \longrightarrow$ const. These conditions are as follows:

$$
\begin{aligned}
\lim _{D a \longrightarrow \infty, \mathrm{Re}_{t} \longrightarrow \infty}\left(\frac{D a}{\mathrm{Re}_{t}}\right) & =\text { const }, \\
\lim _{D a \longrightarrow \infty, \mathrm{Re}_{t} \rightarrow \infty}\left(\frac{\delta_{L}}{\eta}\right) & =0,
\end{aligned}
$$

where $\operatorname{Re}_{t}=u^{\prime} L / v$ is the turbulent Reynolds number (in which the kinematical viscosity coefficient $v$ and molecular transfer coefficient $\chi$ are of the same order, $v \sim \chi), D a=$ $\tau_{t} / \tau_{c h}$ is the Damköhler number (where $\tau_{t}=L / u^{\prime}$ is the turbulent time), and $\eta=L \mathrm{Re}_{t}^{-3 / 4}$ is the Kolmogorov microscale (the size of the minimal eddies). Equation (1b) shows that close to the limit, the size of the minimal eddies $\eta$ remains much larger than the width of the flame $\delta_{L}$. This indicates that the influence of the stretch and curvature of the flame on its structure and speed caused by turbulence is negligible; i.e., in this limiting case, the speed $S_{L}=$ const is not an assumption, but an exact result.

In this case, the probability density function (PDF) of the progress variable is bimodal: $p(c)=P_{u} \delta(c)+P_{b} \delta(1-c)$, where $P_{u}=1-\bar{c}$ and $P_{b}=\bar{c}$ are the probabilities of unburned and burned gases, respectively. The symbol $\delta$ denotes the Dirac generalized function, and the values of the progress variable are $c=0$ for unburned and $c=1$ for burned gases.

\subsection{The Two-Fluid Mathematical Model and Premixed Com-} bustion in the Laminar Flamelet Regime. The laminar flamelet regime arises in the case of developed turbulence $\left(\operatorname{Re}_{t}>>1\right)$, when the thickness of the laminar flame is much less than the size of the smallest eddies, $\delta_{L}<\psi \eta$. Since $L \gg \delta_{L}$ in this case, instantaneous combustion occurs in a highly wrinkled, thin laminar flame, and the application of the two-fluid mathematical model is justified. We rewrite the inequality $\eta / \delta_{L}>1$ using the expressions for $\delta_{L}, \eta$ and the definition of the Damköhler number, as follows:

$$
D a^{1 / 2} \operatorname{Re}_{t}^{-1 / 4}>>1 \text {. }
$$

This inequality shows that in order for the instantaneous flame to be laminar, the Damköhler number must be very large, even in the case of smaller Reynolds numbers. For example, for values of $\mathrm{Re}_{t}=10^{2}$ and $\mathrm{Re}_{t}=10^{3}$ the LHS inequalities become $D a^{1 / 2}>>3$ and $D a^{1 / 2}>>6$. At smaller Damköhler numbers, the laminar flamelet regime does not exist, and the question of the applicability of the two-fluid approach and the corresponding criteria need special consideration.

\subsection{The Two-Fluid Mathematical Model and Combustion in} the Microturbulent Flamelet Regime. In the case of smaller Damköhler numbers for which the width of the laminar flame becomes larger than the sized of minimal turbulent eddies, $\delta_{L}>\eta$, the smaller eddies penetrate the flame, intensifying the transport processes and thickening the flame. If this thickening is not very large, so that the instantaneous microturbulent flame remains strongly curved and the probability of the intermediate states $0<c<1$ is small, a twofluid mathematical model will be applicable to this case. We now obtain the necessary conditions using our theoretical results for the parameters of the thickened (microturbulent) flame, as reported in [16]. These results served as the basis for the TFC turbulent premixed combustion model [17], which we use in this paper to model the mean chemical source appearing in the two-model conditionally averaged equations. 
The speed $U_{f}$, width $\delta_{f}$, and microturbulent transfer coefficient $\chi_{f}$ of the thickened flamelet are described by the following expressions (Eq. (1.6) in [16] and Eq. (3) in [17]):

$$
\begin{gathered}
U_{f} \sim u^{\prime} \cdot D a^{-1 / 2}, \\
\delta_{f} \sim L \cdot D a^{-3 / 2}, \\
\chi_{f} \sim D_{t} \cdot D a^{-2},
\end{gathered}
$$

which were obtained on the assumption that the microturbulent transport inside the thickened flame is controlled by turbulence at statistical equilibrium with a " $k-5 / 3$ " spectrum.

The speed of the turbulent flame is $U_{t}=U_{f}\left(\bar{S} / S_{0}\right)_{f}$, where $\left(\bar{S} / S_{0}\right)_{f}$ is the dimensionless area of the thickened flamelet sheet. Since $\left(\bar{S} / S_{0}\right)_{f}>>1$ (meaning that the flamelet sheet is strongly wrinkled) and $U_{t} \sim u^{\prime}$ (the speed of the developed turbulent premixed flame is of an equal order of magnitude to the characteristic velocity fluctuation), it follows that $U_{f}<<u^{\prime}$. From this and the inequality $\delta_{f}>>\eta$, we can derive a criterion for the existence of a combustion regime in which the microturbulent flamelet sheet remains relatively thin $\left(\delta_{f}<<L\right)$ and strongly wrinkled $\left(U_{t}>>U_{f}\right)$ (Eqs. (1.10) and (1.11) in [16] and Eq. (7) in [17]):

$$
D a^{1 / 2}>>1>>D a^{3 / 2} \mathrm{Re}_{t}^{-3 / 4} \text {. }
$$

We now modernize this criterion slightly, keeping in mind that thickening of the instantaneous flame takes place when $\delta_{L} / \eta>1$, i.e., when $\left(\delta_{L} / \eta\right)^{2}=K a>>1$, where $K a$ is the Karlovitz number. When the condition for strong wrinkling of the thickened flamelet sheet by large-scale eddies, $L / \delta_{f} \sim$ $D a^{1 / 2}>>1$, is added, the altered criterion is as follows:

$$
D a^{1 / 2}>>1>K^{-1} \sim D a \cdot \mathrm{Re}_{t}^{-1 / 2} .
$$

These two criteria for the existence of a strongly wrinkled thickened flamelet sheet are similar in meaning and are satisfied at large Reynolds and moderately large Damköhler numbers.

Example. For $=10, \mathrm{Re}_{t}=10^{3},(4)$ and (5) become $3>>1>>$ 0.2 and $3>>1>>0.2$; for $\mathrm{Da}=30, \mathrm{Re}_{t}=10^{4},(4)$ and (5) become $5.5>>1>>0.016$ and $5.5>>1>>0.3$.

We now estimate the probability of the intermediate states, $0<c<1$. We assume that the speed and width of the turbulent flame are $U_{t} \approx u^{\prime}$ and $\delta_{t} \approx 10 L$, and hence $\left(\bar{S} / S_{0}\right)=U_{t} / U_{f} \approx 3$. The ratio of the volume per unit area of the flamelet sheet and the turbulent flame is $\left(\bar{S} / S_{0}\right) \delta_{f} \approx$ $3 L \cdot D a^{-3 / 2}$ and $\approx 10 L$, giving a small characteristic value of the probability of finding a flamelet sheet in the flame: $P_{f} \approx 3 \cdot D a^{-3 / 2} \approx 10^{-2}$.

We see that the two-fluid approach is approximately applicable to the thickened flamelet combustion regime when the values of the Damköhler number are sufficiently large, $D a \gg 1$. For smaller Damköhler numbers, $D a \geq 1$, at which the preheat zone becomes thicker and less wrinkled, and especially for small numbers, $D a<1$, direct application of the two-fluid approach becomes less suitable.
In [16], a physical explanation was given for why the thickened flamelet, which occurs when $\eta<\delta_{L}$, remains thin, meaning that there is no successive involvement of turbulent eddies of all sizes in the instantaneous flame and a distributed combustion regime is achieved. When its structure reaches statistical equilibrium, flamelet thickening reaches a limit in which "the heat fluxes in the front because of heat conduction and convection, and heat liberation because of chemical reaction have one order of magnitude" (see Eq. (1.7) in [16]).

Even in the case of premixed flames subjected to extreme levels of turbulence studied in a recent paper [18], the instantaneous reaction layer remains thin, continuous, and strongly wrinkled:

"Unlike the preheat zones, which grew exponentially with increasing values of $u^{\prime} / S_{L}$ (up to $\sim 10$ times their laminar value), the reaction layers in all 28 cases study here remained relatively thin, not exceeding 2 times their respective measured laminar values, even though the turbulence level $\left(u^{\prime} / S_{L}\right)$ increased by a factor of $\sim 60[\ldots]$ The reaction layers are also observed to remain continuous; that is, local extinction events are rarely observed".

These experimental data show that a distributed reaction zone is not observed and that accounting for pressure-driven effects can be important for a proper description of the flame even in cases of very strong turbulence. The reaction layers in all 28 cases studied here remained relatively thin, not exceeding two times their respective measured laminar values, even though the turbulence level $\left(u^{\prime} / S_{L}\right)$ increased by a factor of " $\sim 60$ ". We notice that the preheat zones were investigated in [18] using laser induced fluorescence of formaldehyde. Obtained images of the preheat zones do not allow us to find the density gradient that is the relevant quantity for the estimation of a characteristic width of the instantaneous flame. The characteristic width can be significantly smaller than what the images show keeping in mind the very small thickness of the instantaneous reaction zone. The applicability of the two-fluid approximation to the turbulent premixed flames subjected to extreme levels of turbulence remains an open question.

\section{Unclosed Favre-Averaged and Two-Fluid Conditional Equations: Formulation of the Problem}

In this section, we consider two systems of unclosed equations for a turbulent premixed flame in the context of the Favre averaging and two-fluid conditional averaging frameworks; compare their potentialities and limitations; and formulate problems arising in the context of the two-fluid framework.

3.1. Unclosed Favre-Averaged Equations and the Challenge of Countergradient Turbulent Diffusion. The Favre-averaged combustion and hydrodynamics equations for premixed combustion are as follows:

$$
\frac{\partial \bar{\rho} \widetilde{c}}{\partial t}+\nabla \cdot(\bar{\rho} \widetilde{\vec{u}} \widetilde{c})+\nabla \cdot \overrightarrow{\rho \vec{u}}^{\prime \prime} c^{\prime \prime}=\overline{\rho W},
$$




$$
\begin{aligned}
& \bar{\rho}=\frac{\rho_{u}}{\left[1+\widetilde{c}\left(\rho_{u} / \rho_{b}-1\right)\right]}, \\
& \frac{\partial \vec{\rho} \widetilde{\vec{u}}}{\partial t}+\nabla \cdot(\stackrel{\widetilde{\rho} \overrightarrow{\vec{u}}}{\vec{u}})+\nabla \cdot \overline{\rho \vec{u}^{\prime \prime} \vec{u}^{\prime \prime}}=-\nabla \bar{p}, \\
& \frac{\partial \bar{\rho}}{\partial t}+\nabla \cdot \bar{\rho} \widetilde{\vec{u}}=0 .
\end{aligned}
$$

The Favre-averaged value of a variable $a$ is $\widetilde{a}=\overline{\rho a} / \bar{\rho}$ and its instantaneous value is $a=\tilde{a}+a^{\prime \prime}$, where the notation $\bar{a}$ identifies Reynolds averaging, the mean density $\bar{\rho}=\rho_{u} P_{u}+$ $\rho_{b} P_{b}$ and $\bar{\rho} \widetilde{c}=\overline{\rho c}=\rho_{b} P_{b}, P_{u}$ and $P_{b}$ are the probabilities of the unburned and burned gases $\left(P_{u}+P_{b}=1\right)$. The term $\overrightarrow{\rho \vec{u}^{\prime \prime} c^{\prime \prime}}$ in the combustion equation (6a) is a scalar flux for which the $i$-component is $\overline{\rho u_{i}^{\prime \prime} c^{\prime \prime}}$. In the momentum hydrodynamics equations (6c), $\overrightarrow{\rho \vec{u}} \vec{u}^{\prime \prime}$ is a tensor for which the $i j-$ component is $\vec{\rho}_{i}^{\prime \prime} \vec{u}_{j}^{\prime \prime}$. In (6b) for the mean density, $\rho_{u}$ and $\rho_{b}$ are the densities of the unburned and burned gases.

The variables defined for the system in (6a), (6b), (6c), and $(6 \mathrm{~d})$ are the mean density $\bar{\rho}$ and pressure $\bar{p}$, the Favreaveraged progress variable $\widetilde{c}$, and speed $\widetilde{\vec{u}}$. The unknowns in the modeling are the scalar flux (the mean flux of the progress variable) $\overline{\rho \vec{u}} c^{\prime \prime} c^{\prime \prime}$, the stress tensor $\tau=-\overline{\rho \vec{u}^{\prime \prime} \vec{u}^{\prime \prime}}$, and the mean chemical source (the mass consumption rate of the unburned gas per unit volume), which in the case of the flame surface regime is $\overline{\rho W}=\rho_{u} S_{L} \Sigma_{f}$, where $S_{L}$ is the speed of the instantaneous flame relative to the unburned gas and $\Sigma_{f}$ is the flame surface density (the mean area of the instantaneous flame per unit volume). The mass equation (6d) does not contain unknown terms.

"The use of Favre averaging was initially introduced to reduce the system of transport equations to a form equivalent to the case of constant density flows and then using the same turbulent closures, for example, gradient transport with eddy diffusivity modeling of second order transport correlation". The challenge of modeling the scalar flux and stress tensor in the turbulent premixed flame arises from the fact that the former is unusual in the turbulent diffusion countergradient direction, and the latter is abnormally large for the turbulent flow velocity fluctuations described by the diagonal terms of the tensor. As mentioned in the introduction, the gas dynamics nature of these phenomena was clearly explained by Pope in [3].

It is unlikely that the phenomena of the countergradient scalar flux and abnormally large velocity fluctuations in the turbulent premixed flame, caused by the different pressuredriven acceleration of the unburned and burned gases, can be modeled adequately in the context of the Favre averaging paradigm, even using an advanced approach based on attracting the unclosed differential equations for the scalar flux and stresses, and even though these equations involving $\overline{\rho u_{i}^{\prime \prime} c^{\prime \prime}}$ and $\overline{\rho u_{i}^{\prime \prime} u_{i}^{\prime \prime}}$ implicitly contain this hydrodynamic mechanism. The point is that closure approximations for these equations in the context of the Favre averaging framework, where the unknowns are expressed in terms of the Favre-averaged parameters defined by the equations of the problem, do not provide adequate modeling of the hydrodynamic pressuredriven effect. This type of closure is performed in the same manner as that commonly used in turbulence theory; the scalar flux and stresses are presented in the model equations as turbulent phenomena. As an illustration of this shortcoming, we will refer to the results of calculations of the scalar flux on the axis of the impinging premixed flame presented in [19], in which a model theory of this type was developed for the mean fluxes and stresses. The results of the numerical simulations performed in [4] (Figures 5 and 6 in [4]) show a qualitatively different behavior of the pressure profiles on the axis of the flame. In the flame close to the wall (Figure 5 in [4]) the pressure increases slightly across the flame due to the strong influence of the wall on the pressure profile, meaning that in this case, the scalar flux cannot be countergradient. In the free-standing impinging flame, where influence of the wall is small, the strong pressure drops across the flame where $0<\widetilde{c}<1$ (Figure 6 in [4]) can yield a countergradient scalar flux. At the same time, the numerically simulated scalar fluxes presented in these figures are in both cases countergradient and close in value, suggesting that the Favre averaging paradigm does not provide a theoretically adequate description of the effects caused by the different pressuredriven acceleration of the unburned and burned gases.

The reason for this drawback is that the closure of these equations is performed in the context of the Favre averaging framework, in which the unknowns are expressed in terms of the Favre-averaged parameters described by the equations of the problem, and does not adequately model the hydrodynamic pressure-driven effect described without modeling in the context of a conditional averaging paradigm. This type of closure is performed in the same way as that commonly used in the turbulence theory; that is, the scalar flux is interpreted as a turbulent phenomenon. The closure used in [4] allowed the authors to show agreement between their results (using a corresponding empirical constant) and known experimental data for the free-standing flame where the scalar flux was countergradient (Figure 4 in [4]). However, the results of numerical modeling with these empirical constants for the impinging flame close to the wall were not physically feasible, even in a qualitative sense.

The results of numerical simulations of the impinging flame presented in [19] show a gradient scalar flux in the flame close to the wall, and a predominantly countergradient flux in the free-standing flame. In these simulations, the mean scalar flux was estimated as a sum of the terms that describe the countergradient hydrodynamic contribution due to the differences in pressure-driven acceleration of the heavier unburned and lighter burned gases, and the gradient contribution due to turbulent diffusion. We discuss these results in the appendix.

3.2. The Two-Fluid Equations and Modeling Challenges. The conservation equations for the unclosed two-fluid mass (7a), (7b), momentum (7c), (7d), and progress variable were formulated in [8] (Eqs. (23)-(26a) and (26b)) and (17), as follows:

$$
\frac{\partial\left[\rho_{u}(1-\bar{c})\right]}{\partial t}+\nabla \cdot\left[\rho_{u}(1-\bar{c}) \overline{\vec{u}}_{u}\right]=-\rho_{u} S_{L u} \Sigma_{f},
$$




$$
\begin{aligned}
& \frac{\partial\left(\rho_{b} \bar{c}\right)}{\partial t}+\nabla \cdot\left(\rho_{b} \overline{\bar{c}}_{b}\right)=\rho_{b} S_{L b} \Sigma_{f} \\
& \left.\frac{\partial\left[\rho_{u}(1-\bar{c}) \overline{\vec{u}}_{u}\right]}{\partial t}+\nabla \cdot\left[\rho_{u}(1-\bar{c}) \overline{\vec{u}}_{u} \overline{\vec{u}}_{u}\right)\right]+\nabla \\
& \cdot\left[\rho_{u}(1-\bar{c})\left(\overline{\vec{u}}^{\prime} \vec{u}^{\prime}\right)_{u}\right]=-\nabla\left[(1-\bar{c}) \bar{p}_{u}\right] \\
& -\overline{\vec{u}}_{s u} \rho_{u} S_{L u} \Sigma_{f}+(\overline{\vec{p}})_{s u} \Sigma_{f} \\
& \left.\frac{\partial\left[\rho_{b} \overline{\vec{c}}_{b}\right]}{\partial t}+\nabla \cdot\left[\rho_{b} \overline{\vec{c}}_{b} \overline{\vec{u}}_{b}\right)\right]+\nabla \\
& \cdot\left[\rho_{b} \bar{c}\left(\overline{\vec{u}^{\prime} \vec{u}^{\prime}}\right)_{b}\right]=-\nabla\left(\bar{c} \cdot \bar{p}_{b}\right)+\overline{\vec{u}}_{s b} \rho_{b} S_{L b} \Sigma_{f} \\
& -(\overline{p \vec{n}})_{s b} \Sigma_{f} \\
& \frac{\partial \bar{c}}{\partial t}+\overline{\vec{u}}_{s u} \cdot \nabla \bar{c}=\left(\overline{\vec{u}}_{s u}^{\prime} \vec{n}^{\prime}\right)_{s u} \Sigma_{f}+S_{L u} \Sigma_{f},
\end{aligned}
$$

where the indexes " $u$ " and " $b$ " refer to the unburdened and burned gases, respectively; $S_{L u}$ and $S_{L b}$ are the displacement speeds of the instantaneous flame surface relative to the unburned and burned gases $\left(S_{L u}=S_{L}\right.$ using a common notation); $\Sigma_{f}$ is the flame surface density (FSD), the mean area of the instantaneous flame per unit volume; and $\vec{n}$ is the unit normal vector on the flame surface toward the unburned side. The mean progress variable $\bar{c}$ is equal to the probability of the burned gas $P_{b}: \bar{c}=c_{u} P_{u}+c_{b} P_{b}$, where the values of the progress variable are $c_{u}=0$ and $c_{b}=1$, i.e., $P_{b}=\bar{c}$.

In our analysis, we will use the mean chemical source $\overrightarrow{\rho W}(\vec{x}, t)=\dot{m} \Sigma_{f}(\vec{x}, t)$ rather than the FSD $\Sigma_{f}(\vec{x}, t)$, where $\dot{m}=\rho_{u} S_{L u}=\rho_{b} S_{L b}$ is the mass flux per unit area of the instantaneous flame. We can therefore designate the RHS terms in (7a) and (7b) as $-\overline{\rho W}$ and $+\overline{\rho W}$, the second RHS terms in (7c) and (7d) as as $-\overline{\vec{u}}_{s u} \overline{\rho W}$ and $+\overline{\vec{u}}_{s b} \overline{\rho W}$, and the final RHS term in (7e) as $\overline{\rho W} / \bar{\rho}$.

The system in (3a), (3b), and (3c) describes the variables $\bar{c}$, $\overline{\vec{u}}_{u}, \overline{\vec{u}}_{b}, \bar{p}_{u}, p_{b}$. The unknowns that need to be modeled are the conditional tensors $\left({\overrightarrow{\vec{u}}{ }^{\prime} \vec{u}^{\prime}}_{)_{u}}\right.$ and $\left(\overline{\vec{u}^{\prime} \vec{u}^{\prime}}\right)_{u}$ (or the conditional Reynolds stress tensors $\tau_{i j, u}=-\rho_{u}\left(\overrightarrow{\vec{u}}^{\prime} \vec{u}^{\prime}\right)_{u}$ and $\tau_{i j, b}=$ $\left.-\rho_{b}\left(\overrightarrow{\vec{u}}^{\prime} \vec{u}^{\prime}\right)_{u}\right)$, the mean chemical source $\overline{\rho W}$, and the surfaceaveraged variables $\overline{\vec{u}}_{s u},(\overline{p \vec{n}})_{s u}, \overline{\vec{u}}_{s b},(\overline{p \vec{n}})_{s b}$, and $\left(\overline{\vec{u}}_{s u}^{\prime} \vec{n}^{\prime}\right)_{s u}$ defined on the surfaces in the unburned and burned gases adjacent to the instantaneous flame, respectively.

In contrast to the Favre averaging paradigm, the mean scalar flux $\overline{\rho \vec{u}^{\prime \prime} c^{\prime \prime}}$ does not need to be modeled, as it is described by the following closed expression:

$$
\overline{\rho \vec{u}^{\prime \prime} c^{\prime \prime}}=\bar{\rho} \widetilde{c}(1-\widetilde{c})\left(\overline{\vec{u}}_{b}-\overline{\vec{u}}_{u}\right),
$$

where $\bar{\rho}$ and $\widetilde{c}$, in terms of the variable $\bar{c}$, are as follows:

$$
\begin{aligned}
\bar{\rho} & =\rho_{u}(1-\bar{c})+\rho_{b} \bar{c}, \\
\tilde{c} & =\frac{\bar{c}}{\left[\bar{c}+\left(\rho_{u} / \rho_{b}\right)(1-\bar{c})\right]} .
\end{aligned}
$$

We can see that the phenomenon of the countergradient scalar flux in the turbulent premixed flame does need to be modeled, since $\overline{\vec{u}}_{u}, \overline{\vec{u}}_{b}$, and $\bar{c}$ are described by the unclosed system in (7a), (7b), (7c), (7d), and (7e).

The components of the tensor $\overline{\rho \vec{u}} \bar{u}$ are described by the following expression:

$$
\begin{aligned}
\overline{\rho u_{i}^{\prime \prime} u_{j}^{\prime \prime}}= & \bar{\rho}(1-\widetilde{c})\left(\overline{u_{i}^{\prime} u_{j}^{\prime}}\right)_{u}+\bar{\rho} \widetilde{c}\left(\overline{u_{i}^{\prime} u_{j}^{\prime}}\right)_{b} \\
& +\bar{\rho} \widetilde{c}\left(\bar{u}_{i, b}-\bar{u}_{i, u}\right)\left(\bar{u}_{j, b}-\bar{u}_{j, u}\right) .
\end{aligned}
$$

Eq. (10) shows that the "abnormally large velocity fluctuations in the turbulent premixed flame" mentioned above, which are described by the diagonal terms of the tensor $\overline{\rho \vec{u}} \bar{u}$, are controlled by the differences in the mean conditional velocities described directly by the unclosed system in (7a), (7b), (7c), (7d), and (7e).

We proceed on the premise that the conditional averaging framework is likely to be more convenient in the long run than the Favre averaging framework, since the twofluid equations more adequately describe the pressure-driven hydrodynamic processes in the premixed flame caused by a large difference in the densities of unburned and burned gases. Given the possibility of practical use of the two-fluid approach, we identified a critical obstacle in the fundamental difficulty of modeling of the surface mean variables, i.e., their expression in terms of the variables described by the system. At the same time, we proceeded based on the possibility of theoretical modeling of the unknown conditional Reynolds stresses and mean chemical source by modifying existing modeling approaches in the turbulence and combustion theories for our case.

Our formulation of the problem for this theoretical study follows from these considerations.

3.3. Formulation of the Problem. When we began to address this problem, we noticed a connection between the structures of the Favre-averaged mass and moment equations (6d) and (6c), and the two-fluid conditionally averaged mass and moment equations (7a), (7b) and (7c), (7d), which influenced the formulation of the problem. By comparing the mass equations (6d) and (7a), (7b), we can see that the LHS of each conditional mass equation for the unburned (7a) and burned (7b) gases has the structure of the Favre-averaged equation (6d), but is expressed in terms of the conditional variables. The LHS of each conditional equation is weighted by the probabilities of the unburned $\left(P_{u}=(1-\bar{c})\right)$ and burned $\left(P_{b}=\bar{c}\right)$ gas, respectively. Each equation has sink and source terms on the RHS, which are denoted as $-\overline{\rho W}$ and $+\overline{\rho W}$, where the chemical source $\overline{\rho W}$ is the same as in the Favre-averaged equation (6a). A similar connection exists 
between the structures of the momentum equations (6c) and (7c), (7d), where the sink and source represented by the two last terms in $(7 \mathrm{c}),(7 \mathrm{~d})$ express the impulse exchange between the unburned and burned gases.

In the derivation proposed by Lee and Huh [8], generalized functions were used in intermediate, rather cumbersome mathematical manipulations and disappeared in the final conditional equations. The authors used zone averaging (as reflected in the title of the article) and did not actually rely on the two-fluid mathematical model. Since cumbersome calculations using an intermediate value of the progress variable that is nonexistent in the two-fluid mathematical model, $1<c^{*}<1$, led to the physically obvious result mentioned above, a simple method for obtaining these equations by splitting the Favre-averaged mass and momentum equations must therefore exist.

We therefore formulate the research problems considered in this paper as follows:

(1) A simple and physically insightful slipping method for the derivation of the two-fluid conditional mean equations, which allows us to formulate alternative unclosed two-fluid conditionally averaged momentum equations (using sound physical reasoning) that do not contain the surface-averaged unknowns; to formulate unclosed two-fluid equations for the conditional Reynolds stresses; and to reformulate the Favreaveraged equations of the " $k-\varepsilon$ " turbulence model in terms of the mean conditional variables.

(2) A derivation using an analysis of the splitting method of the two-fluid conditional equation in terms of the conditional Reynolds stresses and their analysis.

(3) An unclosed equation for the mean chemical source in the context of the two-fluid approach.

(4) Practical approaches to modeling turbulence in the unburned and burned gases in the context of a twofluid version of the standard " $k-\varepsilon$ " turbulence model, and the mean chemical source using a hypothesis of statistical equilibrium of the small-scale structures.

In preparation for analyzing and resolving the listed tasks in further sections, we aim to do the following:

(i) To remind readers of the two-fluid one-dimensional analytical theory of the countergradient phenomenon developed by the present authors in [15] and to formulate a criterion for the transition from countergradient to gradient scalar flux in the premixed flame.

(ii) To illustrate these results using the examples of impinging and Bunsen flames considered in [19] and [15], respectively.

Consideration of these results yields strong arguments in the favor of the two-fluid conditional averaging framework. We present these materials in the appendix, since although they are not required in order to read the subsequent sections of this article, they not only can help the reader to understand the problem more deeply, but also may be of interest in their own right.

\section{A Splitting Method and Alternative Derivation of the Known Conditional Equations}

In this section, we consider the original derivation splitting method proposed in [9] and demonstrate its effectiveness by rederiving the known zone conditionally averaged mass and momentum equations reported in [8]. We notice that the derivation in [8] is rather cumbersome due to the use of generalized functions and a particular value of the progress variable $0<c^{*}<1$ in the intermediate mathematical manipulations, which disappear from the final unclosed conditionally averaged equations. Our derivation is more direct and simple.

In the next section, we derive alternative conditionally averaged momentum equations using the splitting method, in which the only unknowns are $\left(\overrightarrow{\vec{u}}^{\prime} \vec{u}^{\prime}\right)_{u},\left({\overrightarrow{u^{\prime}} \vec{u}^{\prime}}_{)_{b}}\right.$, and $\overline{\rho W}$.

4.1. The Original Splitting Method. The central idea of this approach is simple: we split the Favre-ensemble-averaged mass (6d) and momentum (6c) equations into averaged twofluid mass and momentum equations for the unburned and burned gases, respectively, using the obvious identity

$$
\overline{\rho a}=\rho_{u} \bar{a}_{u} P_{u}+\rho_{b} \bar{a}_{b} P_{b}=\rho_{u} \bar{a}_{u}(1-\bar{c})+\rho_{b} \bar{a}_{b} \bar{c}
$$

(where $a$ may be a constant, scalar, vector, or tensor variable). We then use the conservation laws for the instantaneous flame to determine the sink and source terms appearing in the two-fluid conditional mass and momentum equations.

4.2. Conditionally Averaged Mass Equations. To transform (6d) using (11), we use $a=1$ and $a=\vec{u}$. This results in the following equation:

$$
\begin{gathered}
\left\{\frac{\partial\left[\rho_{u}(1-\bar{c})\right]}{\partial t}+\nabla \cdot\left[\rho_{u}(1-\bar{c}) \overline{\vec{u}}_{u}\right]\right\}_{u} \\
+\left\{\frac{\partial\left(\rho_{b} \bar{c}\right)}{\partial t}+\nabla \cdot\left(\rho_{b} \overline{\bar{c}}_{b}\right)\right\}_{b}=0
\end{gathered}
$$

where the expressions in the brackets \{\}$_{u}$ and \{\}$_{b}$ refer to reactants and products, respectively. We can easily check whether the expression in the second braces is equal to \{\}$_{b}=$ $\bar{\rho} \widetilde{W}$. To do this, we must eliminate $\widetilde{\vec{u}}$ from (1a) using (6a) and the obvious expressions:

$$
\begin{aligned}
& \overline{\rho c}=\overline{\rho c}=\rho_{b} \bar{c}, \\
& \widetilde{\vec{u}}=\overline{\vec{u}}_{u}(1-\widetilde{c})+\overline{\vec{u}}_{b} \widetilde{c} .
\end{aligned}
$$

Using (12) then gives \{\}$_{\mathcal{u}}=-\overline{\rho W}$, and the conditional mass equations are as follows:

$$
\begin{array}{r}
\frac{\partial\left[\rho_{u}(1-\bar{c})\right]}{\partial t}+\nabla \cdot\left[\rho_{u}(1-\bar{c}) \overline{\vec{u}}_{u}\right]=-\overline{\rho W} \\
\frac{\partial\left(\rho_{b} \bar{c}\right)}{\partial t}+\nabla \cdot\left(\rho_{b} \overline{\vec{c}}_{b}\right)=\overline{\rho W} .
\end{array}
$$


(14a), (14b) are identical to the two-fluid mass equations (23) and (24) in [8].

4.3. Conditionally Averaged Momentum Equations. In a similar way to the analysis in the previous subsection, we present the Favre-averaged momentum equations (6c) as a sum of two groups of terms, which contain conditionally averaged parameters referring to the unburned and burned gases. To obtain these, we insert into (6c) the expressions yielded by (11) with $a=\vec{u}$ and $a=\vec{u} \vec{u}$. This results in the following equation:

$$
\begin{aligned}
& \left\{\frac{\partial\left[\rho_{u}(1-\bar{c}) \overline{\vec{u}}_{u}\right]}{\partial t}+\nabla \cdot\left[\rho_{u}(1-\bar{c}) \overline{\vec{u}}_{u} \overline{\vec{u}}_{u}\right)\right]+\nabla \\
& \left.\cdot\left[\rho_{u}(1-\bar{c})\left(\overline{\vec{u}^{\prime} \vec{u}^{\prime}}\right)_{u}\right]\right\}_{u}+\left\{\frac{\partial\left(\rho_{b} \bar{c} \bar{u}_{i, b}\right)}{\partial t}+\nabla\right. \\
& \left.\cdot\left(\rho_{b} \overline{\vec{c}}_{b} \overline{\vec{u}}_{b}\right)+\nabla \cdot\left(\rho_{b} \bar{c}\left(\overline{\vec{u}^{\prime} \vec{u}^{\prime}}\right)_{b}\right)\right\}_{b} \\
& =-\nabla\left[(1-\bar{c}) \bar{p}_{u}\right]-\nabla\left(\bar{c} \bar{p}_{b}\right),
\end{aligned}
$$

where $\left.\left(\overline{\vec{u}^{\prime} \vec{u}^{\prime}}\right)_{u}, \overline{\left(\vec{u}^{\prime} \vec{u}^{\prime}\right.}\right)_{b}$ and $\bar{p}_{u}, \bar{p}_{b}$ are conditionally averaged moments and pressures. Splitting the equation in (15) yields the following two-fluid momentum equations:

$$
\begin{aligned}
& \left.\frac{\partial\left[\rho_{u}(1-\bar{c}) \overline{\vec{u}}_{u}\right]}{\partial t}+\nabla \cdot\left[\rho_{u}(1-\bar{c}) \overline{\vec{u}}_{u} \overline{\vec{u}}_{u}\right)\right]+\nabla \\
& \cdot\left[\rho_{u}(1-\bar{c})\left(\overline{\vec{u}^{\prime} \vec{u}^{\prime}}\right)_{u}\right]=-\nabla\left[(1-\bar{c}) \bar{p}_{u}\right]+\vec{F}_{u}, \\
& \left.\frac{\partial\left[\rho_{b} \bar{c} \overline{\vec{u}}_{b}\right]}{\partial t}+\nabla \cdot\left[\rho_{b} \bar{c} \overline{\vec{u}}_{b} \overline{\vec{u}}_{b}\right)\right]+\nabla \\
& \cdot\left[\rho_{b} \bar{c}\left(\overline{\vec{u}^{\prime} \vec{u}^{\prime}}\right)_{b}\right]=-\nabla\left(\bar{c} \bar{p}_{b}\right)+\vec{F}_{b}
\end{aligned}
$$

where $\vec{F}_{u}$ and $\vec{F}_{b}$ are equal in value and oppositely directed $\left(\vec{F}_{u}+\vec{F}_{b}=0\right)$ terms that express the impulse sink and source due to the mass exchange between the unburned and burned gases, and the inequality of the pressure on the different sides of the instantaneous flame surface. The equation $\vec{F}_{u}+\vec{F}_{b}=$ 0 expresses the conservation of the averaged impulse on the instantaneous flame surface. The equation

$$
\overline{\vec{u}}_{s u} \overline{\rho W}-(\overline{p \vec{n}})_{s u} \Sigma_{f}=\overline{\vec{u}}_{s b} \overline{\rho W}-(\overline{p \vec{n}})_{s b} \Sigma_{f}
$$

follows from the instantaneous impulse conservation law

$$
\vec{u}_{s u} \dot{m}-p_{s n} \vec{n}=\vec{u}_{b u} \dot{m}-p_{s b} \vec{n}
$$

where $\vec{n}$ is the unit vector normal to the instantaneous flame surface. As we shall see below, the left-hand side and righthand side of the equation (18) are equal, respectively, to $\vec{F}_{u}$ and $\vec{F}_{b}$.

We represent the RHS of (6c) as follows:

$$
\begin{gathered}
-\nabla \bar{p}=-\nabla\left((1-\bar{c}) \bar{p}_{u}\right)-\nabla\left(\bar{c} \bar{p}_{b}\right) \\
+\left\{\left(\overline{\vec{u}}_{s b} \overline{\rho W}-(\overline{p \vec{n}})_{s b} \Sigma_{f}\right)\right. \\
\left.-\left(\overline{\vec{u}}_{s u} \overline{\rho W}-(\overline{p \vec{n}})_{s u} \Sigma_{f}\right)\right\}
\end{gathered}
$$

where the expression in the brackets \{\} is equal to zero in accordance with (17). After splitting the two-fluid momentum equations, (6c) with the RHS presented by (19) yields

$$
\begin{aligned}
& \left.\frac{\partial\left[\rho_{u}(1-\bar{c}) \overline{\vec{u}}_{u}\right]}{\partial t}+\nabla \cdot\left[\rho_{u}(1-\bar{c}) \overline{\vec{u}}_{u} \overline{\vec{u}}_{u}\right)\right]+\nabla \\
& \cdot\left[\rho_{u}(1-\bar{c})\left(\overline{\vec{u}}^{\prime} \vec{u}^{\prime}\right)_{u}\right]=-\nabla\left[(1-\bar{c}) \bar{p}_{u}\right] \\
& -\overline{\vec{u}}_{s u} \overline{\rho W}+(\overline{p \vec{n}})_{s u} \Sigma_{f} \text {, } \\
& \left.\frac{\partial\left[\rho_{b} \overline{\vec{c}}_{b}\right]}{\partial t}+\nabla \cdot\left[\rho_{b} \overline{\vec{c}}_{b} \overline{\vec{u}}_{b}\right)\right]+\nabla \\
& \cdot\left[\rho_{b} \bar{c}\left(\overline{\vec{u}^{\prime} \vec{u}^{\prime}}\right)_{b}\right]=-\nabla\left(\bar{c} \bar{p}_{b}\right)+\overline{\vec{u}}_{s b} \overline{\rho W} \\
& -(\overline{p \vec{n}})_{s b} \Sigma_{f}
\end{aligned}
$$

which are identical to the conditional averaged momentum equations (25) and (26) in [8] that were derived using generalized functions (see $(7 \mathrm{c})$ and $(7 \mathrm{~d})$ above).

A comparison of (16a), (16b), (20a), and (20b) shows that the sink and source terms in (16a) and (16b) are as follows:

$$
\begin{aligned}
& \vec{F}_{u}=-\overline{\vec{u}}_{s u} \overline{\rho W}+(\overline{p \vec{n}})_{s u} \Sigma_{f}, \\
& \vec{F}_{b}=+\overline{\vec{u}}_{s b} \overline{\rho W}-(\overline{p \vec{n}})_{s b} \Sigma_{f} .
\end{aligned}
$$

To avoid misunderstanding, we should emphasize that the splitting method uniquely determines the conditionally averaged mass equations (14a) and (14b) and momentum equations (20a) and (20b).

The alternative conditional momentum equations without the surface-averaged unknowns, which we formulate in the next section, are based on an original concept of a onestep statistical interpretation of instantaneous combustion and an alternative averaged impulse conservation law corresponding to this concept.

\section{Original Statistical Concept of Premixed Combustion in the Flame Surface Regime}

The surface-averaged terms in (21a) and (21b) describe the momentum exchange between the unburned and burned 


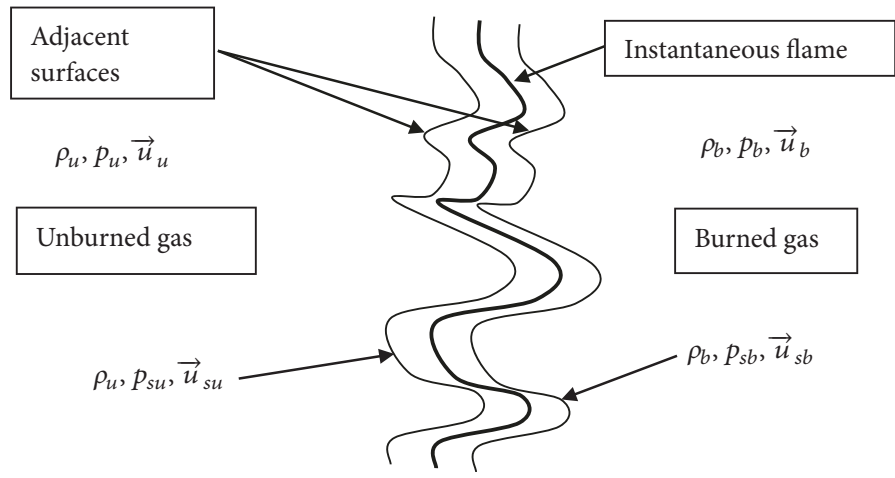

FIGURE 2: Instantaneous parameters in the flow and on the adjacent surfaces.

gases. To avoid the use of the surface-averaged variable, we must express the momentum exchange between the gases in terms of the conditional mean variables. We do this within the framework of the original statistical concept of an instantaneous flame, and we consider this below.

5.1. Statistical Concepts of the One- and Three-Step Processes in Flame Surface Combustion. We consider two statistical concepts of the combustion process in the turbulent premixed flame in the flame surface regime. In accordance with the first concept, the combustion is considered as a global one-step process in which unburned gas with parameters $\rho_{u}, \bar{p}_{u}(\vec{x}, t), \overrightarrow{\vec{u}}_{u}(\vec{x}, t)$ transforms into burned gas with parameters $\rho_{b}, \bar{p}_{b}(\vec{x}, t), \overrightarrow{\vec{u}}_{b}(\vec{x}, t)$, with a rate of transformation equal to $\overrightarrow{\rho W}(\vec{x}, t)$. This global one-step process can be represented schematically as follows:

$$
\rho_{u}, \bar{p}_{u}, \overline{\vec{u}}_{u} \stackrel{\overline{\rho W}}{\Longrightarrow} \rho_{b}, \bar{p}_{b}, \overline{\vec{u}}_{b}
$$

In accordance with the second concept, the global process is split into three subprocesses; these correspond to three successive stages and are represented as follows:

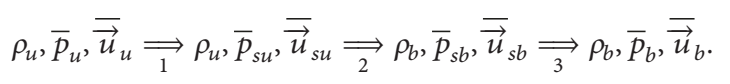

The second stage corresponds to the stepwise transformation of the unburned gas, located on the surface adjacent (infinitely close) to the instantaneous flame, to burned gas, appearing on the infinitely close adjacent surface on the other side of the flame. In the first and third stages, the change in the averaged variables takes place in the unburned and burned gases, respectively.

It is important to keep in mind that the averaged parameters $\bar{p}_{u}, \overline{\vec{u}}_{u}, \bar{p}_{s u}, \overline{\vec{u}}_{s u}$ and $\bar{p}_{b}, \overline{\vec{u}}_{b}, \bar{p}_{s b}, \overline{\vec{u}}_{s b}$ are defined at every point $(\vec{x}, t)$ of the turbulent premixed flame, while the instantaneous parameters $p_{u}, \vec{u}_{u}$ and $p_{b}, \vec{u}_{b}$, are defined in the unburned and burned gases, respectively, and the instantaneous parameters $p_{s u}, \vec{u}_{s u}$ and $p_{s b}, \vec{u}_{s b}$ are defined on the corresponding surfaces adjacent to the instantaneous flame, as shown in Figure 2.
In the general case, the conditional and surface-averaged variables in the unburned and burned gases are not equal, i.e., $\bar{p}_{u} \neq \bar{p}_{s u}, \overline{\vec{u}}_{u} \neq \overline{\vec{u}}_{s u}$ and $\bar{p}_{b} \neq \bar{p}_{s b}, \overline{\vec{u}}_{s b} \neq \overline{\vec{u}}_{b}$. The surface-averaged velocity $\overline{\vec{u}}_{s b}$ of the products directly generated by the instantaneous flame is not necessarily equal to the conditional mean velocity of the burned gas $\overline{\vec{u}}_{b}$. Thus, the interaction of these "newborn" volumes of products with the main flow of the products generates the pressure gradient in the rear zone adjacent to the instantaneous flame surface, giving rise to the equalization of the velocities $\overline{\vec{u}}_{s b} \Longrightarrow \overline{\vec{u}}_{b}$ in the third stage. Similarly, the velocities $\overline{\vec{u}}_{u}$ and $\overline{\vec{u}}_{s u}$ are not necessarily equal due to the pressure gradient generated by combustion in the front zone, which yields the transition $\overrightarrow{\vec{u}}_{u} \Longrightarrow \overline{\vec{u}}_{s u}$.

The flamelet sheet separates the gases with conditional mean parameters $\overline{\vec{u}}_{u}, \bar{p}_{u}$ and $\overline{\vec{u}}_{b}, \bar{p}_{b}$; that is, this sheet includes the instantaneous flame surface and several adjacent layers of unburned and burned gases in which the transitions $\overline{\vec{u}}_{u} \Longrightarrow \overline{\vec{u}}_{s u}$ and $\overline{\vec{u}}_{s b} \Longrightarrow \overline{\vec{u}}_{b}$ take place. In our mathematical analysis, the width of this flamelet sheet is assumed to be zero.

We draw the reader's attention to an analogy between the two statistical concepts of the flamelet combustion described above and two classical theoretical concepts of a detonation wave:

(i) In the Zel'dovich-Neumann-Doering (ZND) theory, a detonation wave is a shock wave followed by a combustion front, i.e., a global process of transformation of cold reactants into hot products that involves two stages: compression of the unburned gas in the shock wave and its chemical transformation into burned gas at the combustion front. Thus, the intermediate parameters of the compressed reactants appear in the equations of the ZND theory.

(ii) In the Chapman-Jouguet (C-J) theory, a detonation wave is considered to be a surface that divides the unburned and burned gases. This surface includes the shock wave and combustion front. Hence, the intermediate parameters of the gas do not appear in the equations of the C-J theory, which include only 
the initial parameters of the reactants and the final parameters of the products.

Using this analogy, we can say that the momentum equations containing intermediate surface-averaged variables (derived by Lee and Huh in [8]) correspond conceptually to the ZND theory, whereas our alternative momentum equations, which do not contain intermediate surface-averaged variables (see (27a) and (27b) below), correspond to the C-J theory.

5.2. Impulse Conservation Law Corresponding to the Two Concepts of the Flame Sheet. The impulse conservation law (17) that was used for formulation of the sink and source terms on the LHS of (20a) and (20b) corresponds to the second concept of the flamelet sheet. It refers to the second stage in (23), in which the unburned gas flow crossing the instantaneous flame becomes the burned gas. The problem of estimating the unknown surface-averaged parameters appearing in this case is reduced to one of modeling the effects of the hydrodynamic mechanisms in the unburned and burned gases that control the transformations of the gas parameters $\underline{\vec{u}}_{u}, \bar{p}_{u} \Longrightarrow \overline{\vec{u}}_{s u}, \bar{p}_{s u}$ and $\overline{\vec{u}}_{s b}, \bar{p}_{s b} \Longrightarrow \overline{\vec{u}}_{b}, \bar{p}_{b}$ in the first and third stages.

We formulate the impulse conservation law for this sheet as follows:

$$
\overline{\vec{u}}_{u} \overline{\rho W}-\bar{p}_{u} \overline{\vec{n}} \Sigma_{f}=\overline{\vec{u}}_{b} \overline{\rho W}-\bar{p}_{b} \overline{\vec{n}} \Sigma_{f}
$$

where $\overline{\vec{n}}(\vec{x}, t)$ is the averaged unit vector normal to the instantaneous flame surface. The terms $\overline{\vec{u}}_{u} \overline{\rho W}$ and $\overline{\vec{u}}_{b} \overline{\rho W}$ describe the sink and source of the impulse per unit volume caused by transformation of the unburned gas (with mean speed $\overline{\vec{u}}_{u}$ ) into the burned gas (with mean speed $\overline{\vec{u}}_{b}$ ), while the terms $-\bar{p}_{u} \overline{\vec{n}} \Sigma_{f}$ and $-\bar{p}_{b} \overline{\vec{n}} \Sigma_{f}$ describe the sink and source due to differences between the mean pressures $\bar{p}_{u}$ and $\bar{p}_{b}$ in the unburned and burned gases. Eq. (24) does not contain a term describing the correlation between the instantaneous pressures $p_{u}$ and $p_{b}$ and the unite vector $\vec{n}$, since these pressures refer to the unburned and burned gases, while the unit vector $\vec{n}$ shows the local orientation of the instantaneous flame surface. We remind the reader that this correlation is significant in (17), since the pressures $p_{s u}$ and $p_{s b}$ are defined on adjacent surfaces that are infinitely close to the instantaneous flame with local orientations described by the unit vector $\vec{n}$.

In essence, the originality of our theoretical analysis consists of using a novel concept of a one-step conversion of unburned gas with conditional mean parameters $\overline{\vec{u}}_{u}, \bar{p}_{u}$ into burned gas with parameters $\overline{\vec{u}}_{b}, \bar{p}_{b}$. This allows us to avoid the appearance of intermediate unknown surface-averaged parameters in the conditionally averaged momentum equations.

It seems that it is impossible to exclude surface-averaged parameters from the exact equation (17), meaning that there is no mathematical equivalence between (17) in terms of surface-averaged parameters and (24) in terms of conditional mean parameters. We therefore consider (24) as an approximation till a possible more rigorous proof. It should be noted that (24) retains original physical meaning also in the case of the thickened flamelet regime, in contrast to (17), where the surface-averaged variables that are defined on the surfaces adjacent to the instantaneous flame surface may cease to be strictly defined. Below we shall validate this equation against a result, known in the literature, from direct numerical simulation (DNS) of the one-dimensional premixed flame. In answer to possible objections from purists, we note that full mathematical strictness of the unclosed equations is not obligatory, since any turbulence and combustion model that could be used for estimation of the unknown conditional Reynolds stresses and mean chemical source appearing in the unclosed equations would inevitably be approximate.

In order to better understand the relationship between these equations, we use an analogy based on the result from turbulence theory that gives an exact formula for the mean dissipation rate $\varepsilon=(\rho v / 2) \sum_{i, j} \overline{\left(\partial u_{i}^{\prime} / \partial x_{j}+\partial u_{j}^{\prime} / \partial x_{i}\right)^{2}}$ in terms of the velocity derivatives. This result is controlled by smallscale turbulence; the formula for the dissipation rate in strong turbulence $\left(\operatorname{Re}_{t}>>1\right)$ expressed in terms of the largescale turbulent parameters $\varepsilon=A u^{\prime 3} / L$ (where $A \sim 1$ is an empirical constant) does not follow from the previous formula, but is a consequence of Kolmogorov's hypothesis of statistical equilibrium in the small-scale structure of turbulence. Although the exact formula and its approximate treatment cannot be mathematically equivalent, the scientific community consider Kolmogorov's expression for the dissipation rate to be a theoretical result of turbulence mechanics.

Analogously, we consider the approximation in (24), which fortunately does not contain an empirical constant, as an approximate impulse conservation law for the instantaneous flame in the turbulent region at high Reynolds numbers (i.e., developed turbulence), large Damköhler numbers $\mathrm{Da}>1$ (i.e., fast chemistry), and large density ratios $\rho_{u} / \rho_{b}>>1$ (i.e., a strong pressure-driven effect). In contrast to the exact equation in (17), this equation is expressed in terms of the conditionally averaged parameters that are described by the conditional mass equations (14a) and (14b) and formulated in the alternative momentum equations (27a) and (27b) below.

5.3. Accuracy of the Alternative Impulse Conservation Law. We have not attempted to estimate the accuracy of (24) with respect to the global parameters of the turbulent flame. We do not expect high accuracy at constant density, $\rho_{u} / \rho_{b}=$ 1 , where both sides of (17) are identical. At the same time, the conditional mean speeds in (24) are not equal in the general case: $\overline{\vec{u}}_{u} \neq \overline{\vec{u}}_{b}$ and $\bar{p}_{u} \neq \bar{p}_{b}$ (the former difference controls the scalar flux in (7a) and affects the stresses in (7b)). Absent DNS results would indicate the extent to which the difference in the velocities in the case of constant density is compensated for by the inequality of the conditional mean pressures, $\bar{p}_{u} \neq \bar{p}_{b}$. The DNS results obtained in [20] for a steady-state, planar premixed flame with density ratio 7.53 and Damköhler number 18.1 demonstrate that, in this case, the exact and approximate equations are fairly close. In order 
to estimate the terms in the exact impulse equation (17), the pressure fluctuation correlations $\left(\overline{p^{\prime} \vec{n}^{\prime}}\right)_{s u}$ and $\left.\overline{\left(p^{\prime} \vec{n}^{\prime}\right.}\right)_{s u}$ are ignored, and the terms containing pressure are presented in the forms $(\overrightarrow{p \vec{n}})_{s u} \Sigma_{f} \approx-\bar{p}_{s u} \nabla \bar{c}$ and $(\overrightarrow{p \vec{n}})_{s b} \Sigma_{f} \approx-\bar{p}_{s b} \nabla \bar{c}$. The authors calculated these expressions, which using our notation become $-\left(\overline{\vec{u}}_{s u}-\overline{\vec{u}}_{u}\right) \overline{\rho W}-\left(\bar{p}_{s u}-\bar{p}_{u}\right) \nabla \bar{c}$ and $\overline{\vec{u}}_{s b}-$ $\left.\overline{\vec{u}}_{b}\right) \overline{\rho W}-\left(\bar{p}_{s b}-\bar{p}_{b}\right) \nabla \bar{c}$. These formulas approximately express the differences in the RHS and LHS terms of (17) and (24), respectively. The graph for the former expression, given in Figure 4 of [20] for the range $\bar{c}=(0.1-0.65)$, shows that the value of this expression is close to zero over the whole range. The graph in Figure 6 of [20] for the latter expression presented for the range $\bar{c}=(0.3-0.98)$ shows that the value of this expression is nearly zero in the range $\bar{c}=(0.3-0.5)$, becomes negative and decreases relatively slowly in the range $\bar{c}=(0.5-0.85)$, and then increases to zero in the range $\bar{c}=$ $(0.85-0.98)$. In general, these results support the conclusion that the exact and approximate equations (17) and (23) are close.

We note that the authors of [20] proposed the use of approximation described above for the terms $(\overrightarrow{p \vec{n}})_{s u} \Sigma_{f}$ and $(\overrightarrow{p \vec{n}})_{s b} \Sigma_{f}$ in modeling conditionally averaged momentum equations that do not contain unknown surface-averaged terms. The pressure-related terms in these equations differ from the corresponding terms in the momentum equations that are obtained in the next section, using a statistical onestep model for transformation in the premixed flame of the unburned gas (with parameters $\rho_{u}, \overline{\vec{u}}_{u}, \bar{p}_{u}$ ) into burned gas (with the parameters $\rho_{b}, \overline{\vec{u}}_{b}, \bar{p}_{b}$ ).

It should be mentioned that this DNS has been performed for the turbulent premixed flame with a relatively low Reynolds number. We have no numerical data for the validation of (24) in the case of much higher Reynolds number where the interaction between hot and cold volumes can be more significant and the difference in the conditional mean velocities lower.

\section{Alternative Momentum Equations and Complete System of Unclosed Equations}

In this section, we obtain conditionally averaged momentum equations without unknown surface-averaged terms and formulate the complete system of unclosed equations as a basis for modeling, as used in further sections.

\subsection{Formulation of the Alternative Conditional Momentum}

Equations. To find $\vec{F}_{u}$ and $\vec{F}_{b}$ in (16a) and (16b), which yield the alternative momentum equations, we represent the RHS of (6a) as follows:

$$
\begin{aligned}
- & \nabla \bar{p} \\
= & -\nabla\left((1-\bar{c}) \bar{p}_{u}\right)-\nabla\left(\bar{c} \bar{p}_{b}\right) \\
& +\left\{\left(\overline{\vec{u}}_{b} \overline{\rho W}-\bar{p}_{b} \overline{\vec{n}} \Sigma_{f}\right)-(\overline{\vec{u}}\right. \\
& \left.\left.\overline{\rho W}-\bar{p}_{u} \overline{\bar{n}} \Sigma_{f}\right)\right\},
\end{aligned}
$$

where the expression in the braces is equal to zero in accordance with (24). After splitting the expressions for $\vec{F}_{u}$ and $\vec{F}_{b}$, (25) yields

$$
\begin{aligned}
& \vec{F}_{u}=\frac{-\left(\overline{\vec{u}}_{u} \overline{\rho W}-\bar{p}_{u} \overline{\vec{n}} \Sigma_{f}\right)}{\sin k}, \\
& \vec{F}_{b}=\frac{\left(\overline{\vec{u}}_{b} \overline{\rho W}-\bar{p}_{b} \overline{\vec{n}} \Sigma_{f}\right)}{\text { source }}
\end{aligned}
$$

Hence, the desired alternative momentum equations are as follows:

$$
\begin{gathered}
\left.\frac{\partial\left[\rho_{u}(1-\bar{c}) \overline{\vec{u}}_{u}\right]}{\partial t}+\nabla \cdot\left[\rho_{u}(1-\bar{c}) \overline{\vec{u}}_{u} \overline{\vec{u}}_{u}\right)\right]+\nabla \\
\cdot\left[\rho_{u}(1-\bar{c})\left(\overline{\vec{u}}^{\prime} \overrightarrow{\vec{u}}^{\prime}\right)_{u}\right]=-\nabla\left[(1-\bar{c}) \bar{p}_{u}\right] \\
-\overline{\vec{u}}_{u} \overline{\rho W}+\bar{p}_{u} \overline{\vec{n}} \Sigma_{f}, \\
\left.\frac{\partial\left[\rho_{b} \overline{\bar{c}_{\vec{u}}}\right]}{\partial t}+\nabla \cdot\left[\rho_{b} \overline{\vec{c}}_{b} \overline{\vec{u}}_{b}\right)\right]+\nabla \\
\cdot\left[\rho_{b} \bar{c}\left(\overline{\vec{u}}^{\prime} \overrightarrow{\vec{u}}^{\prime}\right)\right. \\
\left.\bar{b}_{b}\right]=-\nabla\left(\bar{c} \bar{p}_{b}\right)+\overline{\vec{u}}_{b} \overline{\rho W} \\
-\bar{p}_{b} \overline{\vec{n}} \Sigma_{f} .
\end{gathered}
$$

We eliminate the variable $\Sigma_{f}(\vec{x}, t)$ using the expression $\Sigma_{f}=\overline{\rho W} / \dot{m}$, where $\dot{m}=\rho_{u} S_{L}$. In order to eliminate the unit vector $\overline{\vec{n}}$ from (26a) and (26b), we use (24) to obtain $\overline{\vec{n}} \sim$ $\overrightarrow{\vec{u}}_{u}-\overrightarrow{\vec{u}}_{b}$; i.e., the averaged unit vector $\overrightarrow{\vec{n}}$ is described by (28a):

$$
\begin{aligned}
\overline{\vec{n}} & =\frac{\left(\overline{\vec{u}}_{u}-\overline{\vec{u}}_{b}\right)}{\left|\overline{\vec{u}}_{u}-\overline{\vec{u}}_{b}\right|}, \\
\bar{p}_{u}-\bar{p}_{b} & =\dot{m}\left|\overline{\vec{u}}_{u}-\overline{\vec{u}}_{b}\right| .
\end{aligned}
$$

Combining (28a) with (24) and using the expression $\overline{\rho W}=$ $\dot{m} \Sigma_{f}$ result in (28b), which we will use below. It follows from (28b) that the pressure difference becomes equal to the pressure drop across the laminar flame, $\Delta p=\rho_{u} S_{L}^{2}\left(\rho_{u} / \rho_{b}-1\right)$, at the points of the turbulent flame where the vectors $\overline{\vec{u}}_{u}, \overline{\vec{u}}_{b}$, and $\overline{\vec{n}}$ are collinear.

The reader needs to keep two points in mind: firstly, we regard the unburned and burned gases as incompressible fluids; secondly, the structures of the Favre-averaged equation and corresponding Reynolds-averaged equation of the same problem (which is associated with the constant density approximation) are identical. In our case, these are the momentum equation (6c) and the same equation in which the density is assumed to be constant.

$$
\rho \frac{\partial \overline{\vec{u}}}{\partial t}+\rho \nabla \cdot(\overline{\vec{u} \vec{u}})+\rho \nabla \cdot \overline{\vec{u}^{\prime} \vec{u}^{\prime}}=-\nabla \bar{p}
$$


Hence, the constant density equation (29) also can be used for the formulation of two-fluid conditional equations, leading to (16a) and (16b). We use this consideration in Section 7 when deriving the unclosed two-fluid conditional equations (33a) and (33b) for $\left(\overline{u_{i}^{\prime} u_{j}^{\prime}}\right)_{u}$ and $\left(\overline{u_{i}^{\prime} u_{j}^{\prime}}\right)_{b}$, proceeding from the constant density equation (32) for $\left(\overline{u_{i}^{\prime} u_{j}^{\prime}}\right)$. This trick can be useful when deriving other two-fluid conditional equations.

6.2. Complete System of Unclosed Equations for Turbulent Premixed Combustion. In this section, we summarize the previous results and represent the complete system of unclosed equations, where the unknowns that need to be modeled are the conditionally averaged Reynolds stresses and the mean chemical source. (14a), (14b), (27a), (27b), (28a), and (28b) give the following system of unclosed equations:

$$
\begin{aligned}
& \frac{\partial\left[\rho_{u}(1-\bar{c})\right]}{\partial t}+\nabla \cdot\left[\rho_{u}(1-\bar{c}) \overline{\vec{u}}_{u}\right]=-\overline{\rho W} \\
& \frac{\partial\left(\rho_{b} \bar{c}\right)}{\partial t}+\nabla \cdot\left(\rho_{b} \overline{\bar{c}} \overline{\vec{u}}_{b}\right)=\overline{\rho W} \\
& \left.\frac{\partial\left[\rho_{u}(1-\bar{c}) \overline{\vec{u}}_{u}\right]}{\partial t}+\nabla \cdot\left[\rho_{u}(1-\bar{c}) \overline{\vec{u}}_{u} \overline{\vec{u}}_{u}\right)\right]-\nabla \\
& \cdot\left[(1-\bar{c}) \tau_{u}\right]=-\nabla\left[\left(1-\bar{c}_{)} \bar{p}_{u}\right]\right. \\
& -\left[\overline{\vec{u}}_{u}-\frac{\bar{p}_{u} \dot{m}^{-1}\left(\overline{\vec{u}}_{u}-\overline{\vec{u}}_{b}\right)}{\left|\overline{\vec{u}}_{u}-\overline{\vec{u}}_{b}\right|}\right] \overline{\rho W}, \\
& \left.\frac{\partial\left[\rho_{b} \bar{c} \overline{\vec{u}}_{b}\right]}{\partial t}+\nabla \cdot\left[\rho_{b} \bar{c} \overline{\vec{u}}_{b} \overline{\vec{u}}_{b}\right)\right]-\nabla \\
& \cdot\left[\bar{c}_{b}\right]=-\nabla\left(\bar{c}_{p_{b}}\right) \\
& +\left[\overline{\vec{u}}_{b}-\frac{\bar{p}_{b} \dot{m}^{-1}\left(\overline{\vec{u}}_{u}-\overline{\vec{u}}_{b}\right)}{\left|\overline{\vec{u}}_{u}-\overline{\vec{u}}_{b}\right|}\right] \overline{\rho W}, \\
& \bar{p}_{u}-\bar{p}_{b}=\dot{m}\left|\overline{\vec{u}}_{u}-\overline{\vec{u}}_{b}\right| \cdot
\end{aligned}
$$

The system includes three scalar equations, (30a), (30b), and (30e), and two vector equations, (30c) and (30d). These describe three scalar variables, $\bar{c}, \bar{p}_{u}$, and $\bar{p}_{b}$, and two vector variables, $\overline{\vec{u}}_{u}$ and $\overline{\vec{u}}_{b}$. The unknowns are the conditional stress tensors $\tau_{u}$ and $\tau_{b}$, with components $\tau_{i j, u}=-\rho_{u}\left(\overline{u_{i}^{\prime} u_{j}^{\prime}}\right)_{u}$ and $\tau_{i j, b}=-\rho_{b}\left(\overline{u_{i}^{\prime} u_{j}^{\prime}}\right)_{b}$, and the chemical source $\overline{\rho W}$.

In the context of the two-fluid approach, the terms that describe the scalar flux and stress tensor do not appear in the equations; this means that the challenge of modeling the phenomena of the countergradient scalar flux and abnormal velocity fluctuations in the turbulent flame, which are artifacts of Favre ensemble averaging, does not arise, as mentioned above. At the same time, a quantitative estimate of the mean scalar flux and stresses, and the Reynolds- and Favre-averaged parameters, may be necessary for physical interpretation of the results of numerical simulations and comparison with experimental data. These variables are described by the following exact expressions:

$$
\begin{aligned}
\widetilde{c} & =\frac{\bar{c}}{\left[\bar{c}+\left(\rho_{u} / \rho_{b}\right)(1-\bar{c})\right]}, \\
\widetilde{\vec{u}} & =\vec{u}_{u}(1-\widetilde{c})+\bar{u}_{b} \widetilde{c}, \\
\overline{\vec{u}} & =\vec{u}_{u}(1-\bar{c})+\bar{u}_{b} \bar{c} \\
\bar{\rho} & =\frac{\rho_{u}}{\left[1+\widetilde{c}\left(\rho_{u} / \rho_{b}-1\right)\right]}, \\
\bar{p}= & \bar{p}_{u}(1-\bar{c})+\bar{p}_{b} \bar{c}, \\
\overline{\rho u_{i}^{\prime \prime} c^{\prime \prime}}= & \bar{\rho} \widetilde{c}(1-\widetilde{c})\left(\bar{u}_{i, b}-\bar{u}_{i u}\right), \\
\overline{\rho u_{i}^{\prime \prime} u_{j}^{\prime \prime}=} & \bar{\rho}(1-\widetilde{c})\left(\bar{u}_{i}^{\prime} u_{j}^{\prime}\right)_{u}+\bar{\rho} \widetilde{c}\left(\bar{u}_{i}^{\prime} u_{j}^{\prime}\right)_{b} \\
& +\bar{\rho} \widetilde{c}\left(\bar{u}_{i, b}-\bar{u}_{i u}\right)\left(\bar{u}_{j, b}-\bar{u}_{j u}\right) .
\end{aligned}
$$

(31a), (31b), (31c), (31d), (31e), (31f), and (31g) are not an inherent part of the system in (30a), (30b), (30c), (30d), and (30e), as they are not required for a solution of (30a), (30b), (30c), (30d), and (30e) for given values of $\tau_{i j, u}, \tau_{i j, b}$, and $\overline{\rho W}$. (31a), (31b), (31c), (31d), (31e), (31f), and (31g) are needed to calculate $\widetilde{c}, \widetilde{\vec{u}}, \bar{\rho}, \overline{\rho u_{i}^{\prime \prime} u_{j}^{\prime \prime}}, \overline{\rho u_{i}^{\prime \prime} c^{\prime \prime}}$, and $\bar{p}$ using the values of the variables $\bar{c}, \bar{p}_{u}, \bar{p}_{b}, \overline{\vec{u}}_{u}$, and $\overline{\vec{u}}_{b}$ described by the system in (30a), (30b), (30c), (30d), and (30e), which can be used in the models for the unknown $\tau_{i j, u}, \tau_{i j, b_{b}}$, and $\overline{\rho W}$ and for the interpretation of numerical simulations. We refer to the system that includes (30a), (30b), (30c), (30d), (30e), (31a), (31b), (31c), (31d), (31e), (31f), and (31g) as the complete equation system, as it describes the main Reynolds-, Favre-, and conditionally averaged variables used in applications. We can add equations, for example, formulas for $\bar{T}, \overline{\rho T^{\prime \prime 2}}, \overline{T^{\prime 2}}$, and $\overline{Y_{i}}$, if the temperatures and species concentrations $Y_{i}$ in the unburned and burned gases are known.

We call attention to the absence from the system in (30a), (30b), (30c), (30d), and (30e) of a specific combustion balance equation that is similar to (6a). Eqs. (30a)-(30d) are hydrodynamic mass and momentum equations containing the source $\overline{\rho W}$, meaning that the general problem cannot be broken down into combustion and hydrodynamics subproblems. This does not contradict the initial formulation of the problem, in which (6a), (6b) and (6c), (6d) correspond to the combustion and hydrodynamic subproblems, respectively: the basic Favre-averaged combustion equation (6a) follows from the conditionally averaged mass equations (30a), (30b) and the expression (31f).

In the following two sections, we present unclosed equations for the unknown conditional Reynolds stresses and the mean chemical source, which can form the basis for advanced modeling approaches. In these sections, we propose practical 
approaches for the estimation of these unknowns using a twofluid version of the standard " $k-\varepsilon$ " turbulence model and simple theoretical expressions describing the mean chemical source in the transient turbulent premixed flame observed in the experiments.

The obtained system of the two-fluid equations can be numerically simulated using as a model called "Eulerian Multifluid Modeling" that is embedded in the commercial package Ansys Fluent 6.3 (https://www.sharcnet.ca/Software/ Fluent6/html/ug/node900.htm\#sec-multiphase-eulerian). Fluent's Eulerian multiphase model does not distinguish between fluid-fluid and fluid-solid (granular) multiphase flows. The Fluent solution can be summarized as follows: (i) momentum and continuity equations are solved for each phase (in our case, for the unburned and burned gases); (ii) a single pressure is shared by all phases. The latter condition means that we must set $\bar{p}_{u}=\bar{p}_{b}$ in the two-fluid momentum equations (30c) and (30d) and omit (30e), which is superfluous in this case. This assumption seems permissible from a physical point of view.

It is necessary to stress that we set $\bar{p}_{u}=\bar{p}_{b}$ only due to peculiarities of this commercial package; that is, this assumption is not caused by the essence of the twofluid approach. Note that assuming that conditional mean pressures are equal, strictly speaking, contradicts the basic idea of modeling the momentum interaction between the two fluids. A consequence of this assumption is that the conditional equations (30c) and (30d) lose accuracy (although the Favre-average equation $(6 \mathrm{~d})$ remains exact while being the sum of these inaccurate equations) and therefore the conditional mean speeds as described by those equations are not quite accurate. However, such inaccuracies may be insignificant-as may be indicated indirectly by a comparison of the results, which follow from the simple hydraulic twofluid theory of the countergradient phenomenon-using the assumption of equality of conditionally averaged pressures, with known experimental measurements of the countergradient scalar flux in the turbulent premixed flame; see Appendix A.1.

\section{Unclosed Equations in Terms of Moments}

In this section, we obtain the two-fluid unclosed equations for the unknown conditional moments $\left.\overline{\left(\vec{u} \vec{u}^{\prime}\right.}\right)_{u}$ and $\left(\vec{u}^{\prime} \vec{u}^{\prime}\right)_{b}$. We use the splitting method to formulate the exact equations, which inevitably contain surface-averaged unknowns, and approximate alternative unclosed differential equations that do not contain these unknowns (this derivation is analogous to the use of the splitting method for the two-fluid conditional momentum equations).

We start with the constant density unclosed equations for $\left(\overline{u_{i}^{\prime} u_{j}^{\prime}}\right)$ that are as follows [21] (molecular viscous terms are omitted):

$$
\frac{\partial\left[\rho\left(\overline{u_{i}^{\prime} u_{j}^{\prime}}\right)\right]}{\partial t}+\frac{\partial\left[\rho \bar{u}_{k}\left(\overline{u_{i}^{\prime} u_{j}^{\prime}}\right)\right]}{\partial x_{k}}
$$

$$
\begin{aligned}
= & -\frac{\partial\left[\rho\left(\overline{u_{i}^{\prime} u_{j}^{\prime} u_{k}^{\prime}}\right)\right]}{\partial x_{k}} \\
& -\left[\rho\left(\overline{\bar{u}_{j}^{\prime} u_{k}^{\prime}}\right) \cdot \frac{\partial \bar{u}_{i}}{\partial x_{k}}+\left(\overline{u_{i}^{\prime} u_{k}^{\prime}}\right) \cdot \frac{\partial \bar{u}_{j}}{\partial x_{k}}\right] \\
& -\left[\overline{u_{i}^{\prime} \cdot \frac{\partial p^{\prime}}{\partial x_{j}}}+\overline{u_{j}^{\prime} \cdot \frac{\partial p^{\prime}}{\partial x_{i}}}\right] .
\end{aligned}
$$

Based on the remark at the end of Section 6.1, (32) leads to unclosed conditionally averaged equations for $\left(\overline{u_{i}^{\prime} u_{j}^{\prime}}\right)_{u}$ and $\left(\overline{u_{i}^{\prime} u_{j}^{\prime}}\right)_{b}$ as follows:

$$
\begin{aligned}
& \frac{\partial\left[\rho_{u}(1-\bar{c})\left(\overline{u_{i}^{\prime} u_{j}^{\prime}}\right)_{u}\right]}{\partial t}+\frac{\partial\left[\rho_{u}(1-\bar{c}) \bar{u}_{k, u}\left(\overline{u_{i}^{\prime} u_{j}^{\prime}}\right)_{u}\right]}{\partial x_{k}} \\
& =-\frac{\partial\left[\rho_{u}(1-\bar{c})\left(\overline{u_{i}^{\prime} u_{j}^{\prime} u_{k}^{\prime}}\right)_{u}\right]}{\partial x_{k}} \\
& -\rho_{u}(1-\bar{c})\left[\left(\overline{u_{j}^{\prime} u_{k}^{\prime}}\right)_{u} \frac{\partial \bar{u}_{i, u}}{\partial x_{k}}+\left(\overline{u_{i}^{\prime} u_{k}^{\prime}}\right)_{u} \frac{\partial \bar{u}_{j, u}}{\partial x_{k}}\right] \\
& \left.-(1-\bar{c})\left[\overline{\left(u_{j}^{\prime} \cdot \frac{\partial p^{\prime}}{\partial x_{i}}\right.}\right)_{u}+\left(\overline{u_{i}^{\prime} \cdot \frac{\partial p^{\prime}}{\partial x_{j}}}\right)_{u}\right] \\
& -Q_{i, j}^{u}, \\
& \frac{\partial\left[\rho_{b} \bar{c}\left(\overline{u_{i}^{\prime} u_{j}^{\prime}}\right)_{b}\right]}{\partial t}+\frac{\partial\left[\rho_{b} \bar{c} \bar{u}_{k, b}\left(\overline{u_{i}^{\prime} u_{j}^{\prime}}\right)_{b}\right]}{\partial x_{k}} \\
& =-\frac{\partial\left[\rho_{b} \bar{c}\left(\overline{u_{i}^{\prime} u_{j}^{\prime} u_{k}^{\prime}}\right)_{b}\right]}{\partial x_{k}} \\
& -\rho_{b} \bar{c}\left[\left(\overline{u_{j}^{\prime} u_{k}^{\prime}}\right)_{b} \cdot \frac{\partial \bar{u}_{i, b}}{\partial x_{k}}+\left(\overline{u_{i}^{\prime} u_{k}^{\prime}}\right)_{b} \cdot \frac{\partial \bar{u}_{j, b}}{\partial x_{k}}\right] \\
& \left.-\bar{c}\left[\overline{\left(u_{j}^{\prime} \cdot \frac{\partial p^{\prime}}{\partial x_{i}}\right.}\right)_{b}+\left(\overline{u_{i}^{\prime} \cdot \frac{\partial p^{\prime}}{\partial x_{j}}}\right)_{b}\right]+Q_{i, j}^{b} \text {. }
\end{aligned}
$$

Here $Q_{i j}^{u}$ and $Q_{i j}^{b}$ express the sink and source, $Q_{i j}^{u}=Q_{i j}^{b}$.

7.1. Exact Equations Containing Surface-Averaged Unknowns. We first derive exact expressions for $Q_{i j}^{u}$ and $Q_{i j}^{b}$ in terms of the surface-averaged parameters, which need to be modeled, and then avoid modeling by the formulation of approximate expressions for the sink and source in terms of conditional mean parameters.

We begin the derivation of the exact expressions for $Q_{i j}^{u}$ and $Q_{i j}^{b}$ by formulating the following conservation law for the $i-$ and $j-$ components of the impulse, which connect 
the parameters defined on the surfaces adjacent to the instantaneous flame:

$$
\begin{aligned}
& u_{i, s u}^{\prime} \dot{m}-\left(p_{s u} n_{i}\right)+\overline{p_{s u} n_{i}}=u_{i, s b}^{\prime} \dot{m}-\left(p_{s b} n_{i}\right)+\overline{p_{s b} n_{i}} \\
& u_{j, s u}^{\prime} \dot{m}-\left(p_{s u} n_{j}\right)+\overline{p_{s u} n_{j}} \\
& \quad=u_{j, s b}^{\prime} \dot{m}-\left(p_{s b} n_{j}\right)+\overline{p_{s b} n_{j}},
\end{aligned}
$$

which follow from the instantaneous and averaged impulse equations for the $i$ - component

$$
\begin{aligned}
& \left(\bar{u}_{i, s u}+u_{i, s u}^{\prime}\right) \dot{m}-\left(p_{s u} n_{i}\right) \\
& =\left(\bar{u}_{i, s b}+u_{i, s b}^{\prime}\right) \dot{m}-\left(p_{s b} n_{i}\right), \\
& \bar{u}_{i, s u} \dot{m}-\overline{p_{s u} n_{i}}=\bar{u}_{i, s b} \dot{m}-\overline{p_{s b} n_{i}},
\end{aligned}
$$

and similar equations for the $j$-component. By multiplying the RHS and RHS of (35a) and (35b) and averaging, we obtain the conservation law for the surface-averaged conditional second moments $\left(\overline{u_{i}^{\prime} u_{j}^{\prime}}\right)_{s u}$ and $\left(\overline{u_{i}^{\prime} u_{j}^{\prime}}\right)_{s b}$ as follows:

$$
\begin{aligned}
& \left(\overline{u_{i}^{\prime} u_{j}^{\prime}}\right)_{s u} \dot{m}^{2}-\left[\left(\overline{p u_{i}^{\prime} n_{j}}\right)_{s u}+\left(\overline{p u_{j}^{\prime} n_{i}}\right)_{s u}\right] \dot{m} \\
& \quad+\left[\left(\overline{p^{2} n_{i} n_{j}}\right)_{s u}-\left(\overline{p n_{i}}\right)_{s u}\left(\overline{p n_{j}}\right)_{s u}\right] \\
& =\left(\overline{u_{i}^{\prime} u_{j}^{\prime}}\right)_{s b} \dot{m}^{2}-\left[\left(\overline{p u_{i}^{\prime} n_{j}}\right)_{s b}+\left(\overline{p u_{j}^{\prime} n_{i}}\right)_{s b}\right] \dot{m} \\
& \quad+\left[\left(\overline{p^{2} n_{i} n_{j}}\right)_{s b}-\left(\overline{p n_{i}}\right)_{s u}\left(\overline{p n_{j}}\right)_{s b}\right] .
\end{aligned}
$$

When $\dot{m}=0$ (i.e., there is no combustion), (34a) and (34b) become the identity $0=0$, as all of the mean parameters defined on both adjacent surfaces are equal. For the case $\dot{m}>0$, we divide (36) by $\dot{m}$ and then multiply it by $\Sigma_{f}$; remembering that $\dot{m} \Sigma_{f}=\overline{\rho W}$, we obtain the following equation:

$$
\begin{aligned}
& \left(\overline{u_{i}^{\prime} u_{j}^{\prime}}\right)_{s u} \overline{\rho W}-\left[\left(\overline{p u_{i}^{\prime} n_{j}}\right)_{s u}+\left(\overline{p u_{j}^{\prime} n_{i}}\right)_{s u}\right] \Sigma_{f} \\
& +\left[\frac{\left(\overline{p^{2} n_{i} n_{j}}\right)_{s u}}{\dot{m}}-\frac{\left(\overline{p n_{i}}\right)_{s u}\left(\overline{p n_{j}}\right)_{s u}}{\dot{m}}\right] \Sigma_{f} \\
& =\left(\overline{u_{i}^{\prime} u_{j}^{\prime}}\right)_{s b} \overline{\rho W}-\left[\left(\overline{p u_{i}^{\prime} n_{j}}\right)_{s b}+\left(\overline{p u_{j}^{\prime} n_{i}}\right)_{s b}\right] \Sigma_{f} \\
& +\left[\frac{\left(\overline{p^{2} n_{i} n_{j}}\right)_{s b}}{\dot{m}}-\frac{\left(\overline{p n_{i}}\right)_{s u}\left(\overline{p n_{j}}\right)_{s b}}{\dot{m}}\right] \Sigma_{f} .
\end{aligned}
$$

Hence, the exact sink and source terms in (33a) and (33b) are as follows:

$$
\begin{aligned}
Q_{i j}^{u}= & \frac{\left(\overline{u_{i}^{\prime} u_{j}^{\prime}}\right)_{s u} \overline{\rho W}-\left[\left(\overline{p u_{i}^{\prime} n_{j}}\right)_{s u}+\left(\overline{p u_{j}^{\prime} n_{i}}\right)_{s u}\right] \Sigma_{f}}{1}, \frac{}{2} \\
& +\frac{\left[\left(\overline{p^{2} n_{i} n_{j}}\right)_{s u} / \dot{m}-\left(\overline{p n_{i}}\right)_{s u}\left(\overline{p n_{j}}\right)_{s u} / \dot{m}\right] \Sigma_{f},}{3}
\end{aligned}
$$

$$
\begin{aligned}
Q_{i j}^{b}= & \frac{\left(\overline{u_{i}^{\prime} u_{j}^{\prime}}\right)_{s b} \overline{\rho W}-\left[\left(\overline{p u_{i}^{\prime} n_{j}}\right)_{s b}+\left(\overline{p u_{j}^{\prime} n_{i}}\right)_{s b}\right] \Sigma_{f}}{2} \\
& +\frac{\left[\left(\overline{p^{2} n_{i} n_{j}}\right)_{s b} / \dot{m}-\left(\overline{p n_{i}}\right)_{s b}\left(\overline{p n_{j}}\right)_{s b} / \dot{m}\right] \Sigma_{f}}{3}
\end{aligned}
$$

The terms marked with ' 1 ' describe the sink and source due to the transformation of the unburned gas into burned gas at a rate $\overline{\rho W}$. The terms marked with '2', which do not depend explicitly on $\dot{m}$, represent contributions to the sink and source due to differences in the velocity fluctuations and pressures on the "cold" and "hot" surfaces adjacent to the random instantaneous flame, with an orientation characterized by the unit vector $\vec{n}$. The terms marked with ' 3 ' depend explicitly on $\dot{m}$ and are caused by differences in the pressures on the isosurfaces. All surface-averaged variables are unknowns.

7.2. Alternative Two-Fluid Conditional Equations without Surface-Averaged Unknowns. Eq. (37) corresponds to the second stage in (23). Recall that the processes in stages 1 and 3 do not make contributions to the sink and source terms $Q_{i j}^{u}$ and $Q_{i j}^{b}$, as these processes take place in the nonreacting unburned and burned gases, meaning that (37) describes the global one-stage process denoted by (22) for the surfaceaveraged parameters. To avoid the appearance of surfaceaveraged unknowns, we formulate an approximate one-stage equation in terms of conditional averaged variables (in a similar way to the conditional moment equations analyzed above) as follows:

$$
\begin{aligned}
& \left(\overline{u_{i}^{\prime} u_{j}^{\prime}}\right)_{u} \overline{\rho W}-\left[\left(\overline{p^{\prime} u_{i}^{\prime}}\right)_{u} \bar{n}_{j}+\left(\overline{p^{\prime} u_{j}^{\prime}}\right)_{u} \bar{n}_{i}\right] \Sigma_{f} \\
& +\left[\frac{\overline{p_{u}^{\prime 2}}\left(\bar{n}_{i} \bar{n}_{j}+\overline{n_{i}^{\prime} n_{j}^{\prime}}\right)}{\dot{m}}\right] \Sigma_{f} \\
& =\left(\overline{u_{i}^{\prime} u_{j}^{\prime}}\right)_{b} \overline{\rho W}-\left[\left(\overline{p^{\prime} u_{i}^{\prime}}\right)_{b} \bar{n}_{j}+\left(\overline{p^{\prime} u_{j}^{\prime}}\right)_{b} \bar{n}_{i}\right] \Sigma_{f} \\
& +\left[\frac{\overline{p_{b}^{\prime 2}}\left(\bar{n}_{i} \bar{n}_{j}+\overline{n_{i}^{\prime} n_{j}^{\prime}}\right)}{\dot{m}}\right] \Sigma_{f} .
\end{aligned}
$$

Hence, the approximate sink and source terms in (33a) and (33b), $\widetilde{Q}_{i j}^{u}$ and $\widetilde{Q}_{i j}^{u}$, are as follows:

$$
\begin{aligned}
\widetilde{Q}_{i j}^{u}= & \left(\overline{u_{i}^{\prime} u_{j}^{\prime}}\right)_{u} \overline{\rho W}-\left[\left(\overline{p^{\prime} u_{i}^{\prime}}\right)_{u} \bar{n}_{j}+\left(\overline{p^{\prime} u_{j}^{\prime}}\right)_{u} \bar{n}_{i}\right] \Sigma_{f} \\
& +\left[\frac{\overline{p_{u}^{\prime 2}}\left(\bar{n}_{i} \bar{n}_{j}+\overline{n_{i}^{\prime} n_{j}^{\prime}}\right)}{\dot{m}}\right] \Sigma_{f}=Q_{i j}^{b} \\
= & \left(\overline{u_{i}^{\prime} u_{j}^{\prime}}\right)_{b} \overline{\rho W}-\left[\left(\overline{p^{\prime} u_{i}^{\prime}}\right)_{b} \bar{n}_{j}+\left(\overline{p^{\prime} u_{j}^{\prime}}\right)_{b} \bar{n}_{i}\right] \Sigma_{f} \\
& +\left[\frac{\overline{p_{b}^{\prime 2}}\left(\bar{n}_{i} \bar{n}_{j}+\overline{n_{i}^{\prime} n_{j}^{\prime}}\right)}{\dot{m}}\right] \Sigma_{f} .
\end{aligned}
$$

The conditional mean unknowns that need to be modeled are the terms in square brackets, where the unit vector 
$\vec{n}$ is described by (28a) ( $\overline{\rho W}$ obeys the unclosed equation considered below, and $\left.\Sigma_{f}=\overline{\rho W} / \dot{m}\right)$. In order to develop model equations for the conditional Reynolds stresses, the unknowns in (33a), (33b), and (40) must be expressed in terms of the parameters described by the system (30a), (30b), (30c), (30d), and (30e).

\section{Unclosed Equation in Terms of the Mean Chemical Source}

The most commonly used method for obtaining the unclosed equation describing the mean chemical source is based on the equation $\overline{\rho W}=\rho_{u} S_{L} \Sigma$, where the flame surface density (FSD) $\Sigma$ (the mean area of the instantaneous flame per unit volume) obeys a surface-averaged unclosed $\Sigma$-equation. We showed in [12] that this equation is inconsistent with other Favreaveraged equations (6a), (6b), (6c), and (6d) for the problem. Here, we show that the $\Sigma$-equation is also inconsistent with the system of two-fluid conditional equations (30a), (30b), (30c), (30d), and (30e). In [12], we derived the Favre-averaged unclosed $\widetilde{W}$ - equation, which directly describes the mean chemical source $\overline{\rho W}=\bar{\rho} \widetilde{W}$ and is consistent with the system of Favre-averaged equations (6a), (6b), (6c), and (6d). We reformulate this equation in terms of the conditional mean variables so that it becomes compatible with the system of two-fluid conditional equations (30a), (30b), (30c), (30d), and (30e).

8.1. Incompatibility of the $\Sigma$-Equation with Other Equations. The flame surface density $\Sigma$ is controlled by turbulence, which generates wrinkles in the instantaneous flame, and moves with speed $S_{L}$ relative to the unburned gas, which smoothes these wrinkles. The FSD equations describing the mean chemical source are as follows:

$$
\begin{aligned}
& \overline{\rho W}=\rho_{u} S_{L} \Sigma, \\
& \frac{\partial \Sigma}{\partial t}+\nabla \cdot\left(\overline{\vec{u}}_{s} \Sigma\right)=\left[(\overline{\nabla \cdot \vec{u}})_{s}-(\overline{\vec{n} \vec{n}: \nabla \vec{u}})_{s} \Sigma-\nabla\right. \\
& \quad \cdot\left[(\overrightarrow{S \vec{n}})_{s} \Sigma\right]+(\overline{S \nabla \cdot \vec{n}})_{s} \Sigma,
\end{aligned}
$$

where $\vec{n}=-\nabla c(\vec{x}, t) /|\nabla c(\vec{x}, t)|$ is the unit vector normal to the instantaneous flame, which points toward the reactants, and $\vec{n} \vec{n}: \nabla \vec{u}=n_{i} n_{j} \partial u_{i} / \partial x_{j}$. The "s" symbol represents surface averaging. This equation is defined on a particular isosurface inside the instantaneous flame traveling with speed $S$. While $\Sigma$ is almost the same for all isosurfaces $\rho_{b}<\rho<\rho_{u}$, the surface-averaged velocity $\overline{\vec{u}}_{s}$ and speed $S$ change across the instantaneous flame, on the "cold" isosurface.

Although correct in itself, the surface-averaged equation (41b) is inconsistent not only with the Favre-averaged equations for the problem, as shown in [12], but also with the two-fluid conditional equations (30a), (30b), (30c), (30d), and (30e), due to the appearance of differently averaged speeds $\overline{\vec{u}}_{u}, \overline{\vec{u}}_{b}$, and $\overline{\vec{u}}_{s}$ and other surface-averaged variables that would result in unnecessary and unjustified difficulties in modeling.
8.2. Consistent Unclosed Equation for the Mean Chemical Source. As mentioned in the introduction, the mean chemical source is not a conditionally averaged characteristic, but a statistical characteristic of the traveling flame surface dividing the unburned and burned gases. Hence, the unclosed equation for the mean chemical source, which is compatible with other two-fluid conditional equations, inevitably contains conditional mean variables referring to both the unburned and burned gases. We propose to use the unclosed Favre-averaged equation in terms of $\widetilde{W}$ for the turbulent premixed combustion in the flamelet regime, as derived in [12]:

$$
\begin{aligned}
& \frac{\partial(\bar{\rho} \widetilde{W})}{\partial t}+\nabla \cdot(\bar{\rho} \widetilde{\vec{u}} \widetilde{W})+\nabla \cdot\left(\bar{\rho}\left\langle\vec{u}^{\prime \prime} W^{\prime \prime}\right\rangle_{F}\right) \\
& \quad+\rho_{u} S_{L} \nabla \cdot(\overline{\vec{n} W}) \\
& =\bar{\rho} \widetilde{W}\left\langle\nabla_{t} \cdot \vec{u}\right\rangle_{F}+\bar{\rho}\left\langle W^{\prime \prime}\left(\nabla_{t} \cdot \vec{u}\right)^{\prime \prime}\right\rangle_{F} \\
& \quad+2 \rho_{u} S_{L} \overline{K W}
\end{aligned}
$$

where $\widetilde{W}=\overline{\rho W} / \bar{\rho}, K=0.5 \nabla \cdot \vec{n}$ is the curvature of the instantaneous flame, and the notations $\widetilde{a}=\langle a\rangle_{F}$ and $\left\langle a^{\prime \prime} b^{\prime \prime}\right\rangle_{F}$ indicate Favre averaging.

The LHS of (42) contains one nonstationary term and three transport terms describing convection by (i) mean velocity transport; (ii) turbulent diffusion-type transport due to the correlation of velocity and combustion rate fluctuations; and (iii) transport caused by the instantaneous flame movement. The three terms on the RHS describe the effects of different physical mechanisms (sources and sinks) that control the Favre-averaged $\widetilde{W}$ : (i) the flame stretch due to the mean velocity field; (ii) the flame stretch due to the fluctuation component of the velocity; and (iii) the effect of the wrinkled flame propagation on the sheet area.

In the case of the two-fluid mathematical model analyzed here (i.e., for zero width of the instantaneous flame), there is no flame stretch, as shown in Section 2.1; hence, the first and second RHS terms must be omitted. The unclosed equation for the mean chemical source then becomes

$$
\begin{gathered}
\frac{\partial(\overline{\rho W})}{\partial t}+\nabla \cdot(\overline{\rho W} \widetilde{\vec{u}})+\nabla \cdot\left(\overline{\rho \vec{u}^{\prime \prime} W^{\prime \prime}}\right)+\rho_{u} S_{L} \nabla \\
\cdot(\overline{\vec{n} W})=2 \rho_{u} S_{L} \overline{K W} .
\end{gathered}
$$

where the Favre-averaged velocity $\widetilde{\vec{u}}$ and mean density $\bar{\rho}$ are expressed in terms of $\overline{\vec{u}}_{u}, \overline{\vec{u}}_{b}, \rho_{u}, \rho_{b}$, and $\bar{c}$ by (31a), (31b), and (31d). The unknowns that need to be modeled are in the third and fourth terms on the LHS and the term on the RHS and should be expressed in terms of the variables described by (30a), (30b), (30c), (30d), (30e), (31a), (31b), (31c), (31d), (31e), (31f), and (31g). Equation (43) was not presented in [12]. 


\section{Practical Approaches to Modeling the Conditional Reynolds Stresses}

In this section, we consider this relatively simple approach to modeling the conditional Reynolds stresses $\tau_{i j, u}=-\rho_{u}\left(\overline{u_{i}^{\prime} u_{j}^{\prime}}\right)_{u}$ and $\tau_{i j b,}=-\rho_{b}\left(\overline{u_{i}^{\prime} u_{j}^{\prime}}\right)_{b}$ in the context of the classical " $k-\varepsilon$ " turbulence model [21], reformulated in terms of the two-fluid conditional variables using the splitting method. We present the conditional mean turbulent stresses as follows:

$$
\begin{aligned}
\tau_{i j, u} & =-\rho_{u}\left(\overline{u_{i}^{\prime} u_{j}^{\prime}}\right)_{u} \\
& =v_{t, u}\left(\frac{\partial \bar{u}_{i, u}}{\partial x_{j}}+\frac{\partial \bar{u}_{j, u}}{\partial x_{i}}\right)-\left(\frac{2}{3}\right) \rho_{u} \bar{k}_{u} \delta_{i j}, \\
\tau_{i j b,} & =-\rho_{b}\left(\overline{u_{i}^{\prime} u_{j}^{\prime}}\right)_{b} \\
& =v_{t, b}\left(\frac{\partial \bar{u}_{i, b}}{\partial x_{j}}+\frac{\partial \bar{u}_{j, b}}{\partial x_{i}}\right)-\left(\frac{2}{3}\right) \rho_{u} \bar{k}_{b} \delta_{i j},
\end{aligned}
$$

where $\quad v_{t, u}=C_{\mu} \bar{k}_{u}^{2} / \bar{\varepsilon}_{u}$ and $v_{t, b}=C_{\mu} \bar{k}_{b}^{2} / \bar{\varepsilon}_{b}$ are the conditional turbulent viscosity coefficients $\left(C_{\mu}\right.$ is an empirical coefficient) and $\delta_{i j}$ is the Kronecker delta function.

9.1. Two-Fluid Version of the " $k-\varepsilon$ " Turbulence Model. The conditionally averaged turbulent energies and dissipation rates $\bar{k}_{u}, \bar{\varepsilon}_{u}, \bar{k}_{b}, \bar{\varepsilon}_{b}$ can be described by conditionally averaged equations, which we formulate by splitting the Favreaveraged equations of the standard " $k-\varepsilon$ " turbulence model [21]:

$$
\begin{aligned}
\frac{\partial(\bar{\rho} \tilde{k})}{\partial t}+\frac{\partial\left(\bar{\rho} \tilde{u}_{\alpha} \widetilde{k}\right)}{\partial x_{\alpha}}= & \frac{\tau_{i j} \partial \tilde{u}_{j}}{\partial x_{i}} \\
& +\frac{\partial\left[\bar{\rho}\left(v_{t} / \sigma_{k}\right) \partial \widetilde{k} / \partial x_{i}\right]}{\partial x_{i}} \\
& -\bar{\rho} \widetilde{\varepsilon}, \\
\frac{\partial(\bar{\rho} \widetilde{\varepsilon})}{\partial t}+\frac{\partial\left(\bar{\rho} \tilde{u}_{i} \widetilde{\varepsilon}\right)}{\partial x_{i}}= & \frac{C_{\varepsilon 1} \tau_{i j}(\widetilde{\varepsilon} / \widetilde{k}) \partial \bar{u}_{j}}{\partial x_{i}} \\
& -C_{\varepsilon 2} \bar{\rho}\left(\frac{\widetilde{\varepsilon}^{2}}{\widetilde{k}}\right) \\
& +\partial\left[\frac{\bar{\rho}\left(v_{t} / \sigma_{\varepsilon}\right) \partial \widetilde{\varepsilon}}{\partial x_{i}}\right] \partial x_{i},
\end{aligned}
$$

where $v_{t}=C_{\mu}\left(\tilde{k}^{2} / \widetilde{\varepsilon}\right)$ is the turbulent viscosity coefficient, $C_{\mu}, C_{\varepsilon 1}, C_{\varepsilon 2}, \sigma_{k}, \sigma_{\varepsilon}$ are the empirical coefficients of the standard " $k-\varepsilon$ " turbulence model, and $\tau_{i j}$ are the components of the stress tensor:

$$
\tau_{i j}=-\left(\rho \overline{u_{i}^{\prime \prime} u_{j}^{\prime \prime}}\right)=v_{t}\left(\frac{\partial \tilde{u}_{i}}{\partial x_{j}}+\frac{\partial \tilde{u}_{j}}{\partial x_{i}}\right)-\left(\frac{2}{3}\right) \bar{\rho} \tilde{k} \delta_{i j} .
$$

These equations are as follows:

$$
\begin{aligned}
& \frac{\partial\left[\bar{\rho}(1-\widetilde{c}) \bar{k}_{u}\right]}{\partial t}+\frac{\partial\left[\bar{\rho}(1-\widetilde{c}) \bar{u}_{\alpha, u} \bar{k}_{u}\right]}{\partial x_{\alpha}} \\
& =\frac{\bar{\rho}(1-\widetilde{c})\left(\tau_{i j, u} / \rho_{u}\right) \partial \bar{u}_{i, u}}{\partial x_{j}}-\bar{\rho}(1-\widetilde{c}) \bar{\varepsilon}_{u} \\
& +\frac{\partial\left[\bar{\rho}(1-\tilde{c})\left(v_{t, u} / \sigma_{k}\right) \partial \bar{k}_{u} / \partial x_{i}\right]}{\partial x_{i}}-\underline{\bar{k}_{u} \overline{\rho W}} \\
& +\underline{K_{u} \Sigma_{f}}, \\
& \frac{\partial\left(\bar{\rho} \widetilde{c} \bar{k}_{b}\right)}{\partial t}+\frac{\partial\left(\bar{\rho} \widetilde{c}_{i, b} \bar{k}_{b}\right)}{\partial x_{i}} \\
& =\frac{\bar{\rho} \widetilde{c}\left(\tau_{i j, b} / \rho_{b}\right) \partial \bar{u}_{i, b}}{\partial x_{j}}-\bar{\rho} \widetilde{c \varepsilon}_{b} \\
& +\frac{\partial\left[\bar{\rho} \widetilde{c}\left(v_{t, b} / \sigma_{k}\right) \partial \bar{k}_{b} / \partial x_{i}\right]}{\partial x_{i}}+\underline{\bar{k}_{b} \overline{\rho W}}+\underline{K_{b} \Sigma_{f}}, \\
& \frac{\partial\left[\bar{\rho}(1-\widetilde{c}) \bar{\varepsilon}_{u}\right]}{\partial t}+\frac{\partial\left[\bar{\rho}(1-\widetilde{c}) \bar{u}_{i, u} \bar{\varepsilon}_{u}\right]}{\partial x_{i}} \\
& =\frac{C_{\varepsilon 1} \bar{\rho}(1-\widetilde{c})\left(\bar{\varepsilon}_{u} / \bar{k}_{u}\right)\left(\tau_{i j, u} / \rho_{u}\right) \partial \bar{u}_{i, u}}{\partial x_{j}} \\
& -C_{\varepsilon 2} \bar{\rho}(1-\widetilde{c})\left(\frac{\bar{\varepsilon}_{u}^{2}}{\bar{k}_{u}}\right) \\
& +\frac{\partial\left[\bar{\rho}(1-\widetilde{\mathcal{c}})\left(v_{t, u} / \sigma_{k}\right) \partial \bar{k}_{u} / \partial x_{\alpha}\right]}{\partial x_{\alpha}}-\underline{\bar{\varepsilon}_{u} \overline{\rho W}} \\
& +\underline{E_{u} \Sigma_{f}} \\
& \frac{\partial\left(\bar{\rho} \widetilde{c \varepsilon}_{b}\right)}{\partial t}+\frac{\partial\left(\bar{\rho} \widetilde{c} \bar{u}_{i, b} \bar{\varepsilon}_{b}\right)}{\partial x_{i}} \\
& =\frac{C_{\varepsilon 1} \bar{\rho}(1-\widetilde{c})\left(\bar{\varepsilon}_{b} / \bar{k}_{b}\right)\left(\tau_{i j, b} / \rho_{b}\right) \partial \bar{u}_{i, b}}{\partial x_{j}} \\
& -C_{\varepsilon 2} \bar{\rho} \widetilde{c}\left(\frac{\bar{\varepsilon}_{b}^{2}}{\bar{k}_{b}}\right)+\frac{\partial\left[\bar{\rho} \widetilde{c}\left(v_{t, u} / \sigma_{k}\right) \partial \bar{k}_{b} / \partial x_{\alpha}\right]}{\partial x_{\alpha}} \\
& +\underline{E_{b} \Sigma_{f}}+\underline{\bar{\varepsilon}_{b} \overline{\rho W}} .
\end{aligned}
$$

The underlined terms in the balance equations (47a), (47b), (47c), and (47d) obey the following conditions:

$$
\begin{aligned}
& -\bar{k}_{u} \overline{\rho W}+K_{u} \Sigma_{f}=+\bar{k}_{b} \overline{\rho W}+K_{b} \Sigma_{f}, \\
& -\bar{\varepsilon}_{u} \overline{\rho W}+E_{u} \Sigma_{f}=+\bar{\varepsilon}_{b} \overline{\rho W}+E_{b} \Sigma_{f} .
\end{aligned}
$$

The sink terms $-\bar{k}_{u} \overline{\rho W},-\bar{\varepsilon}_{u} \overline{\rho W}$ and source terms $+\bar{k}_{b} \overline{\rho W}$, $+\bar{\varepsilon}_{b} \overline{\rho W}$ are caused by transformation of the unburned gas 
with turbulent parameters $\bar{k}_{u}$ and $\bar{\varepsilon}_{u}$ into burned gas with turbulent parameters $\bar{k}_{b}$ and $\bar{\varepsilon}_{b}$. The unknown exchange terms, which are symbolically represented as $K_{u} \Sigma_{f}, K_{b} \Sigma_{f}$ and $E_{u} \Sigma_{f}, E_{b} \Sigma_{f}$, describe the effects of the volume expansion of the gas due to combustion on the mean conditional kinetic energies and dissipation rates.

9.2. A Simple Method to Avoid Modeling the Unknown Exchange Terms. To avoid modeling these exchange terms, we took into account the fact that the influence of the expansion due to combustion on turbulence of the unburned gas is relatively small in comparison with its influence on turbulence of the burned gas due to effect of strong turbulization of the gas when it crosses the wrinkled instantaneous flame. Our proposed approach involves the following:

(i) Omission of the terms $K_{u} \Sigma_{f}$ and $E_{u} \Sigma_{f}$ in (47a) and (47c) as negligible.

(ii) Using the equations of the standard " $k-\varepsilon$ " turbulence model instead of conditionally averaged equations (47b) and (47d).

(iii) Estimation of the conditional means $\bar{k}_{b}$ and $\bar{\varepsilon}_{b}$ using two algebraic expressions that involve $\tilde{k}, \bar{k}_{u}, \bar{k}_{b}$ and $\widetilde{\varepsilon}, \bar{\varepsilon}_{u}, \bar{\varepsilon}_{b}$, respectively.

These simplifications lead to the following system of equations:

$$
\begin{aligned}
& \frac{\partial\left[\bar{\rho}(1-\widetilde{\mathcal{c}}) \bar{k}_{u}\right]}{\partial t}+\frac{\partial\left[\bar{\rho}(1-\widetilde{\mathcal{c}}) \bar{u}_{\alpha, u} \bar{k}_{u}\right]}{\partial x_{\alpha}} \\
& =\frac{\bar{\rho}(1-\widetilde{c})\left(\tau_{i j, u} / \rho_{u}\right) \partial \bar{u}_{i, u}}{\partial x_{j}} \\
& +\frac{\partial\left[\bar{\rho}(1-\widetilde{c})\left(v_{t, u} / \sigma_{k}\right) \partial \bar{k}_{u} / \partial x_{i}\right]}{\partial x_{i}}-\bar{\rho}(1-\widetilde{c}) \bar{\varepsilon}_{u} \\
& -\bar{k}_{u} \overline{\rho W}, \\
& \frac{\partial\left[\bar{\rho}(1-\widetilde{c}) \bar{\varepsilon}_{u}\right]}{\partial t}+\frac{\partial\left[\bar{\rho}(1-\widetilde{c}) \bar{u}_{i, u} \bar{\varepsilon}_{u}\right]}{\partial x_{i}} \\
& =\frac{C_{\varepsilon 1} \bar{\rho}(1-\widetilde{c})\left(\bar{\varepsilon}_{u} / \bar{k}_{u}\right)\left(\tau_{i j, u} / \rho_{u}\right) \partial \bar{u}_{i, u}}{\partial x_{j}} \\
& +\frac{\partial\left[\bar{\rho}(1-\widetilde{c})\left(v_{t, u} / \sigma_{k}\right) \partial \bar{k}_{u} / \partial x_{\alpha}\right]}{\partial x_{\alpha}}-C_{\varepsilon 2} \bar{\rho}(1-\widetilde{c}) \\
& \cdot\left(\frac{\bar{\varepsilon}_{u}^{2}}{\bar{k}_{u}}\right)-\bar{\varepsilon}_{u} \overline{\rho W} \\
& \frac{\partial(\bar{\rho} \tilde{k})}{\partial t}+\frac{\partial\left(\bar{\rho} \tilde{u}_{\alpha} \tilde{k}\right)}{\partial x_{\alpha}}=\frac{\tau_{i j} \partial \tilde{u}_{j}}{\partial x_{i}} \\
& +\frac{\partial\left[\bar{\rho}\left(v_{t} / \sigma_{k}\right) \partial \widetilde{k} / \partial x_{i}\right]}{\partial x_{i}}-\bar{\rho} \widetilde{\varepsilon},
\end{aligned}
$$

$$
\begin{aligned}
& \frac{\partial(\bar{\rho} \widetilde{\varepsilon})}{\partial t}+\frac{\partial\left(\bar{\rho} \widetilde{u}_{i} \widetilde{\varepsilon}\right)}{\partial x_{i}}=\frac{C_{\varepsilon 1} \tau_{i j}(\widetilde{\varepsilon} / \widetilde{k}) \partial \bar{u}_{j}}{\partial x_{i}} \\
& \left.\quad+\partial\left[\frac{\bar{\rho}\left(v_{t} / \sigma_{\varepsilon}\right) \partial \widetilde{\varepsilon}}{\partial x_{i}}\right] \partial x_{i}-C_{\varepsilon 2} \bar{\rho}\left(\frac{\widetilde{\varepsilon}^{2}}{\widetilde{k}}\right)\right], \\
& \widetilde{k}=\bar{k}_{u}(1-\widetilde{c})+\bar{k}_{b} \widetilde{c} \\
& \widetilde{\varepsilon}=\bar{\varepsilon}_{u}(1-\widetilde{c})+\bar{\varepsilon}_{b} \widetilde{c},
\end{aligned}
$$

which allow us to avoid estimating the turbulization of the burned gas using the conditional turbulent energy $\bar{k}_{b}$ and dissipation rate $\bar{\varepsilon}_{b}$. This turbulization in terms of $\widetilde{k}$ and $\widetilde{\varepsilon}$ is described to some extent by the Favre-averaged equations (49c) and (49d), and the effect on the values of the conditional $\bar{k}_{b}$ and $\bar{\varepsilon}_{b}$ is described by (49e) and (49f). It should be emphasized that the empirical constants in this modeling approach are the same as in the standard " $k-\varepsilon$ " turbulence model [21].

\section{Simple Modeling of the Mean Chemical Source}

To model the mean chemical source $\overline{\rho W}$, we proceed from the following assumptions:

(1) This source is the same in both the set of unclosed Favre-averaged equations (6a), (6b), (6c), and (6d) and the set of unclosed two-fluid conditional equations (30a), (30b), (30c), (30d), and (30e). This means that we can use models obtained in the context of the Favre averaging framework and only reformulate them in terms of the conditional mean variables described by the two-fluid equations.

(2) The model for the chemical source depends on the combustion regime. We consider the laminar and thickened flamelet regimes, since a distributed regime is not observed in experiment. In these regimes, instantaneous combustion takes place in thin and strongly wrinkled flame sheets, which can be considered as random ones.

(3) The chemical source model also depends on the stage of the turbulent flame. Real turbulent flames are transient, meaning that complete statistical equilibrium is not achieved in strongly wrinkled, random flamelet sheets. We model the chemical source for a transient flame in the intermediate asymptotic stage, where small-scale wrinkles in the flamelet sheet reach statistical equilibrium, while the large-scale wrinkles remain in nonequilibrium. This concept allows us to obtain theoretical results that we will use to formulate valid models of the chemical source.

Following $[16,17,22]$, we show that the flame in the intermediate asymptotic stage is characterized by a constant turbulent speed $U_{t}$ and increasing width $\delta_{t}$. We assume that the one-dimensional turbulent flame travels along the $x$-axis and that the wrinkled surface, which shows the configuration 
of the flamelet sheet, is described by a random single-valued function $x=h(y, z, t)$ with a continuous power spectral density (PSD) $F(k)$. The density is constant, the turbulence is uniform and stationary, and the mean velocity of the medium is zero. The speed of the flame is $U_{t}=S_{L}\left(\bar{A} / A_{0}\right)$, where $\left(\bar{A} / A_{0}\right)>>1$ is the dimensionless mean area of the instantaneous flame. The area in terms of the spectrum is estimated as follows.

$$
\begin{aligned}
\left(\frac{\bar{A}}{A_{0}}\right) & =\overline{\left(1+|\nabla h|^{2}\right)^{1 / 2}} \approx \overline{|\nabla h|} \approx\left(\overline{|\nabla h|^{2}}\right)^{1 / 2} \\
& =\left(\int_{0}^{\infty} k^{2} F(k) d k\right)^{1 / 2}
\end{aligned}
$$

The equality marked with ' 1 ' in (50) is an exact expression for the area of the random surface described by the equation $x=$ $h(y, z, t)$. The transition marked with ' 2 ' is valid for $|\nabla h|^{2}>>$ 1 due to the assumption $\left(\bar{A} / A_{0}\right)>>1$. The transition marked with ' 3 ' is an estimate (an average of the absolute magnitude of a random function with zero mathematical expectation is approximately equal to the square root of its dispersion). The equality marked with ' 4 ' is the exact expression of this root in terms of the spectrum of the random surface $F(k)$. This gives

$$
\begin{aligned}
\left(\frac{\bar{A}}{A_{0}}\right) & \approx\left(\int_{0}^{\infty} k^{2} F(k) d k\right)^{1 / 2}, \\
\sigma_{f}^{2}(t) & =\overline{(x-\bar{x}(t))^{2}}=\int_{0}^{\infty} F(k, t) d k
\end{aligned}
$$

i.e., the small- and large-scale wrinkles (large and small wave numbers $k$ in FSD) control the dimensionless mean area $\left(\bar{A} / A_{0}\right)$ and the dispersion $\sigma_{f}^{2}$ of the instantaneous flame, respectively.

The assumption that the small-scale structures in the transient turbulent flame are already statistical at equilibrium results in a nearly constant speed of the flame $U_{t}=$ $S_{L}\left(\bar{A} / A_{0}\right)$. Small-scale equilibrium is achieved when the rate of generation for small-scale wrinkles in the flamelet sheet by small-scale turbulent eddies with speed $u_{e}^{\prime}$ that is equal to or less than the flamelet speed $\left(u_{e}^{\prime} \leq S_{L}\right.$ for the laminar flamelet regime) and the rate of their consumption by the moving flamelet sheet surface are equal. At the same time, large-scale structures are not yet at equilibrium, resulting in an increase in the flame width. In the case of strong turbulence, when $u^{\prime} \gg S_{L}$, this increase is controlled by turbulent diffusion. The dispersion of the instantaneous flame $\sigma_{f}^{2}$ and the width of the flame in this case are as follows:

$$
\begin{aligned}
\sigma_{f}^{2} & \cong 2 D_{t} t, \\
\delta_{t} & \sim\left(u^{\prime} L t\right)^{1 / 2},
\end{aligned}
$$

where the turbulent diffusion coefficient $D_{t} \sim u^{\prime} L$.

In $[17,22]$, we referred to a flame with this type of statistical structure as an "intermediate steady propagation (ISP) flame". The kinematic equations describing a onedimensional ISP flame in a motionless medium of constant density and a three-dimensional ISP flame in a moving medium are as follows:

$$
\begin{gathered}
\frac{\partial \bar{c}}{\partial t}=\frac{D_{t} \partial^{2} \bar{c}}{\partial x^{2}}+U_{t}\left|\frac{\partial \bar{c}}{\partial x}\right|, \\
\frac{\partial \bar{c}}{\partial t}+\overline{\vec{u}} \nabla \bar{c}=\nabla \cdot\left(D_{t} \nabla \bar{c}\right)+U_{t}|\nabla \bar{c}| .
\end{gathered}
$$

The mean chemical sources in these equations are $\bar{W}=$ $U_{t}|\partial \bar{c} / \partial x|$ and $\bar{W}=U_{t}|\nabla \bar{c}|$. In the case of variable density, the chemical term in the corresponding Favre-averaged equations becomes

$$
\overline{\rho W}=\bar{\rho} \widetilde{W}=\rho_{u} U_{t}|\nabla \widetilde{c}| .
$$

The problem is then reduced to obtaining theoretical expressions for the speed $U_{t}$, which are different for the laminar and thickened flamelet combustion mechanisms. This problem was considered in [22] for the laminar flamelet regime (using the flame surface mathematical model) and in $[16,17]$ for the thickened (microturbulent) regime.

10.1. The Chemical Source for the Laminar Flamelet Regime. In the flamelet regime in the case of constant density and strong turbulence $\left(u^{\prime}>>S_{L}\right)$, the speed and time interval of the ISP flame are described by the following expressions [22]:

$$
\begin{aligned}
& U_{t} \sim\left(u^{\prime} S_{L}\right)^{1 / 2}, \\
& \tau_{t} \leq t<<\left(\frac{u^{\prime}}{S_{L}}\right)^{2} \tau_{t} .
\end{aligned}
$$

We use (55a) in modeling the chemical source in the case when the gas densities and the turbulent characteristics are different for the unburned and burned gases. We assume that the turbulent flame speed $U_{t}$ is controlled by the turbulence of the unburned gas. Hence, the modal expression for the chemical source $(\overline{\rho W})_{L}$ used in the two-fluid conditional equations becomes

$$
(\overline{\rho W})_{L}=A_{L} \rho_{u} \sqrt{\bar{k}_{u}^{1 / 2} S_{L}}|\nabla \widetilde{c}|
$$

where $A_{L} \sim 1$ is an empirical constant, $\widetilde{c}$ is expressed in (31a) in terms of $\bar{c}$, as described by the two-fluid equations, $S_{L}$ is the speed of the laminar flame relative to the unburned gas, and $\bar{k}_{u}=(3 / 2) \overline{u_{u}^{\prime 2}}$ is the turbulent energy in the unburned gas, as described by the two-fluid model equations of turbulence considered in Section 9.

The formula (55a) is also invalid at large times $t>$ $\left(u^{\prime} / S_{L}\right)^{2} \tau_{t}$, when wrinkles of all sizes reach statistical equilibrium. A theoretical analysis of this steady-state flame propagation was carried out in [22] using a hyperbolic equation that directly described the front edge of the flame, controlling its propagation speed, and showed that this speed was $U_{t}=\left(u^{\prime 2}+S_{L}^{2}\right)^{1 / 2}$. Hence, the flame speed at strong turbulence $u^{\prime} / S_{L}>1$ becomes $U_{t} \cong u^{\prime}$, meaning that this analysis confirmed and clarified the known classical 
Damköhler and Shelkin estimate $U_{t} \sim u^{\prime}$. As this theoretical result indicates a paradoxical contradiction of the numerical experimental data (since the speed of the turbulent flame does not depend on the physicochemical properties of the combustible mixture), we referred to it in previous work as the Damköhler-Shelkin paradox [22]. We remind the reader that the steady-state stage is unattainable in practice for real flames.

Strictly speaking, (55a) is also invalid for small times $t \leq \tau_{t}$ when the wrinkles of all sizes are at statistical disequilibrium. Accurate simulations on these timescales are mainly important for spark-ignition engines. We do not consider this case here and make the assumption that (56) and the analogous equation (58) for the thickened flamelet regime are valid at all times. In this regard, it should be noted that the statistical equilibrium of small-scale turbulent structures is postulated in the " $k-\varepsilon$ " turbulence model.

10.2. The Chemical Source for the Thickened Flamelet Regime. The possibility of applying the two-fluid approach in the case of the thickened (microturbulent) flamelet regime was analyzed in Section 2.3. The conditions under which the thickened flamelet sheet remains thin and strongly wrinkled are described by the inequalities in (4) and (5).

The formula for the speed of the constant-pressure transient ISP flame propagating in a uniform flow, obtained in $[16,17]$ (Eq. (2.9) in [16] and Eq. (6) in [17]), and the inequality for the time interval for this transient flame are as follows:

$$
\begin{aligned}
& U_{t}=A u^{\prime} D a^{1 / 4}=A u^{\prime 3 / 4} S_{L}^{1 / 2} \chi^{-1 / 4} L^{1 / 4}, \\
& \tau_{t}<t<<\tau_{t} D a,
\end{aligned}
$$

where $A$ is an empirical coefficient, the Damköhler number $D a=\tau_{t} / \tau_{c h} \gg 1$, the turbulent time $\tau_{t}=L / u^{\prime}$, and the chemical time $\tau_{c h}=\chi / S_{L}^{2}$.

We use (57a) and assume that the turbulent flame speed $U_{t}$ in the case of variable density is controlled by turbulence in the unburned gas, which is characterized by the conditional turbulent energy $\bar{k}_{u}$ and dissipation rate $\bar{\varepsilon}_{u}$, as described by the turbulence model equations. Thus, we formulate the model expression for the mean chemical source $(\overline{\rho W})_{m t}$ for the case of the microturbulent flamelet regime as follows:

$$
(\overline{\rho W})_{m t}=A_{m t} \rho_{u} \bar{k}_{u}^{3 / 4} \bar{\varepsilon}_{u}^{-1 / 4} S_{L}^{1 / 2} \chi_{u}^{-1 / 4}|\nabla \widetilde{c}|,
$$

where the empirical coefficients $A_{m t}$ and $A_{L}$ appearing in the model equations for the laminar and microturbulent flamelet regimes have different values. The molecular heat transfer coefficient $\chi_{u}$ and the speed of the laminar flame are the physicochemical characteristics of the combustible mixture in this case.

\section{Conclusions}

Widely used in applications RANS models of turbulent premixed combustion are based on the use the Favre-averaged equations. There is a large number of papers devoted to different aspects of the RANS approach, some RANS models implemented in commercial codes. The totality of all results obtained in the context of the Favre averaging framework, constitute a certain technological tool widely used in many industries. Nevertheless we are sure that the Favre averaging approach is likely to be less convenient in the long run than two-fluid conditional averaging approach for modelling turbulent premixed combustion in the flamelet regime. The reason is that from a conceptual point of view, the latter approach yields more adequate description of hydrodynamic and turbulent process in the unburned and burned gases, and their mutual interaction. The challenge of modeling of the phenomena of the counter-gradient scalar flux and abnormally large velocity fluctuations in the premixed flame, which, in fact, are treated in the context of Favre average framework as turbulent phenomena ("counter-gradient turbulent diffusion" and "abnormally strong turbulence"), disappear in the context of two-fluid framework. The reason is that these phenomena are controlled by the difference of the conditional mean velocities, which are described directly (without modeling) by the two-fluid conditional equations.

At the same time, the practical use of the two-fluid approach faces significant difficulties. This is due to the appearance in the two-fluid unclosed equations along with requiring modeling conditional Reynolds stresses also surface-averaged unknowns, and the chemical source, which depends on the statistical structure of the instantaneous flame-surface. The unclosed equations become more cumbersome when going beyond the two-fluid model is taken into account the small but finite width of the instantaneous flame [23], which seems unjustified.

We summarize the results of our affords to overcome these difficulties and to propose possible way for resolving arising modeling problem are as follows:

(1) We formulate a system of two-fluid conditionally averaged unclosed equations (26a) and (26b) for turbulent premixed combustion in the flamelet regime, where the only unknowns that need to be modeled are the conditional Reynolds stresses $\tau_{i j, u}=-\rho_{u}\left(\overline{u_{i}^{\prime} u_{b}^{\prime}}\right)_{u}$, $\tau_{i j, b}=-\rho_{b}\left(\overline{u_{i}^{\prime} u_{b}^{\prime}}\right)_{b}$ and the chemical source $\overline{\rho W}=$ $\rho_{u} S_{L} \Sigma_{f}$

(2) To avoid the appearance of unknown surfaceaveraged parameters, which are inevitable in known, mathematically exact, unclosed two-fluid equations, we propose the one-step statistical concept of the flame surface combustion regime, in which every point of the turbulent flame undergoes direct transformation from unburned gas with parameters $\rho_{u}, \bar{p}_{u}, \overline{\vec{u}}_{u}$ to burned gas with parameters $\rho_{b}, \bar{p}_{b}, \overline{\vec{u}}_{b}$.

(3) We develop a simple splitting method (without using generalized functions) that allows us to rederive known [8] equations containing surface-averaged terms and to formulate alternative conditional equations that do not contain these unknown terms. This method is used for the formulation of equations describing the conditional Reynolds stresses and for the reformulation of the equations of the " $k-\varepsilon$ " 
turbulence model in the context of the two-fluid approach.

(4) We then formulate a system of unclosed equations that describes the conditional mean and Reynoldsand Favre-averaged parameters, including the mean scalar flux and stress tensor, which may be necessary for the interpretation of the results of numerical simulations and their comparison with experimental data.

(5) We touch on the problem of modeling the unknown Reynolds stresses $\tau_{i j, u}, \tau_{i j, b}$ and the mean chemical source $\overline{\rho W}$, as follows: (i) we consider unclosed equations in terms of $\left(\overline{u_{i}^{\prime} u_{b}^{\prime}}\right)_{\mathcal{u}},\left(\overline{u_{i}^{\prime} u_{b}^{\prime}}\right)_{b}$, and $\widetilde{W}$, which may be used in developing advanced models, and (ii) we propose a simpler modeling approach in which the Reynolds stresses are estimated in the context of the modified " $k-\varepsilon$ " turbulence model and the chemical source is described by an algebraic expression referring to a transient turbulent flame in the intermediate asymptotic stage.

(6) These unclosed two-fluid conditionally averaged equations and the approaches proposed here for the estimation of appearing unknowns can be treated as an alternative paradigm for modeling turbulent premixed combustion in the flamelet regime.

\section{Appendix}

In this appendix, we consider the phenomenon of the countergradient scalar flux in the turbulent premixed flame, caused by the different pressure-driven acceleration of the heavier unburned and lighter burned gases. An analysis of this hydrodynamic mechanism requires methods that fall outside the scope of the hypothesis-based approach used above. We consider this important problem in the context of our simple hydraulic two-fluid theory of the countergradient phenomenon.

\section{A. The Countergradient Scalar Flux and Its Transition to the Gradient Case}

The hydrodynamic mechanism dominates in the turbulent premixed flame when the fall in pressure across the flame $\Delta p=\rho_{u} U_{t}^{2}\left(\rho_{u} / \rho_{b}-1\right)$ is sufficiently large. This takes place for a large ratio of the densities $\rho_{u} / \rho_{b}>>1$ and strong turbulence, $u^{\prime} / S_{L}>>1$. For estimation purposes, we assume $\rho_{u} / \rho_{b} \sim 10$, $u^{\prime} / S_{L} \sim 10, U_{t} \sim u^{\prime}, \delta_{t} \sim\left(u^{\prime} / S_{L}\right) L \sim 10 L$, where $u^{\prime}$ and $L$ are the characteristic values of the velocity fluctuations and the size of the turbulent eddies. For estimation of the characteristic value $q_{p-d}$ of the pressure-driven component of the scalar flux $\left(\overline{\rho u^{\prime \prime} c^{\prime \prime}}\right)_{p-d}$ using (8), we assume $\bar{u}_{u}=U_{t}$, $\bar{u}_{b}=\left(\rho_{u} / \rho_{b}\right) U_{t} \sim 10 U_{t}$, and $\widetilde{c} \sim 0.5$, giving $q_{p-d} \sim 2 \bar{\rho} U_{t}$. The characteristic value $q_{t . d}$. of the turbulent diffusion component $\left(\overline{\rho u^{\prime \prime} c^{\prime \prime}}\right)_{t . d .}=-\bar{\rho} D_{t} d \tilde{c} / d x$ is $q_{t . d .} \sim \bar{\rho} u^{\prime} L / \delta_{t} \sim 10^{-1} \bar{\rho} u^{\prime}$. Hence, the ratio $q_{p-d} / q_{t . d} \sim 20$, meaning that the scalar flux is controlled by the hydrodynamic effect caused by thermal expansion. The transition from countergradient to gradient scalar flux takes place when this ratio falls to $q_{p-d} / q_{t . d} \sim 1$. In cases where $q_{p-d} / q_{t . d}$. $<<1$, the pressure-driven effect is small, and the scalar flux is gradient.

A.1. Simple Hydrodynamic (Hydraulic) Two-Fluid Theory of the Countergradient Phenomenon. In [15], we formulated the two-fluid mass and momentum equations assuming conservation of the total pressure of the unburned gas across the flame brush, which allowed us to find the mean scalar flux $\left(\rho u^{\prime \prime} c^{\prime \prime}\right)_{p d}$ in the turbulent premixed flame with known speed $U_{t}$ and the ratio $\rho_{u} / \rho_{b}$. In nondimensional form, these dimensionless equations in the coordinate system traveling with the flame front are as follows, using $U_{t}, \rho_{u}$, and $\rho_{u} U_{t}^{2}$ as quantities to normalize the velocity, density, and pressure [15]:

$$
\begin{aligned}
(1-\bar{c}) \bar{u}_{u}+\frac{\bar{c} \bar{u}_{b}}{\Theta} & =1, \\
\bar{p}+(1-\bar{c}) \bar{u}_{u}^{2}+\frac{\bar{c} \bar{u}_{b}^{2}}{\Theta} & =\bar{p}_{-\infty}+1, \\
\bar{p}_{u}+\left(\frac{1}{2}\right) \bar{u}_{u}^{2} & =\bar{p}_{-\infty}+\frac{1}{2},
\end{aligned}
$$

where $\Theta=\rho_{u} / \rho_{b}$ and the mean pressure $\bar{p}$ is given by $\bar{p}=(1-$ $\bar{c}) \bar{p}_{u}+\bar{c} \bar{p}_{b}$. Assuming that the conditional mean pressures are equal, i.e., $\bar{p}_{u}=\bar{p}_{b}$ (hydraulic approximation), (A.1a), (A.1b), and (A.1c) reduce to the following set of equations:

$$
\begin{gathered}
(1-\bar{c}) \bar{u}_{u}+\frac{\bar{c} \bar{u}_{b}}{\Theta}=1, \\
\left(\frac{1}{2}-\bar{c}\right) \bar{u}_{u}^{2}+\frac{\bar{c} \bar{u}_{b}^{2}}{\Theta}=\frac{1}{2},
\end{gathered}
$$

which have the solution [15]:

$$
\begin{aligned}
\bar{u}_{b} & =\frac{-\beta+\left(-4 \alpha \gamma+\beta^{2}\right)^{1 / 2}}{2 \alpha}, \\
\bar{u}_{u} & =\frac{1-\bar{u}_{b} \bar{c} / \Theta}{1-\bar{c}}, \\
\alpha & =\frac{\bar{c}}{\Theta}\left[\frac{0.5-\bar{c}}{(1-\bar{c})^{2}} \frac{\bar{c}}{\Theta}+1\right], \\
\beta & =-2 \frac{\bar{c}}{\Theta} \frac{0.5-\bar{c}}{(1-\bar{c})^{2}}, \\
\gamma & =\frac{0.5-\bar{c}}{(1-\bar{c})^{2}}-0.5 .
\end{aligned}
$$

The dimensionless expressions for the scalar flux and prolonged stress component are as follows.

$$
\begin{aligned}
& \overline{\rho u^{\prime \prime} c^{\prime \prime}}=\bar{\rho} \widetilde{c}(1-\widetilde{c})\left(\bar{u}_{b}-\bar{u}_{u}\right), \\
& \overline{\rho u^{\prime \prime} u^{\prime \prime}}=\bar{\rho} \widetilde{c}\left(\bar{u}_{b}-\bar{u}_{u}\right)^{2}
\end{aligned}
$$

Together with (A.3), (31a) and (31d) yield the exact result without empirical parameters. The dimensionless conditional 


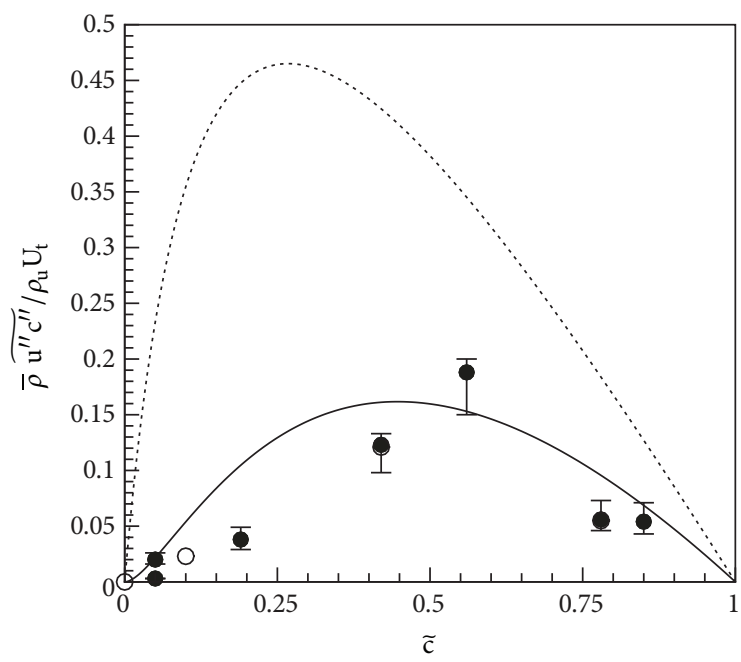

(a)

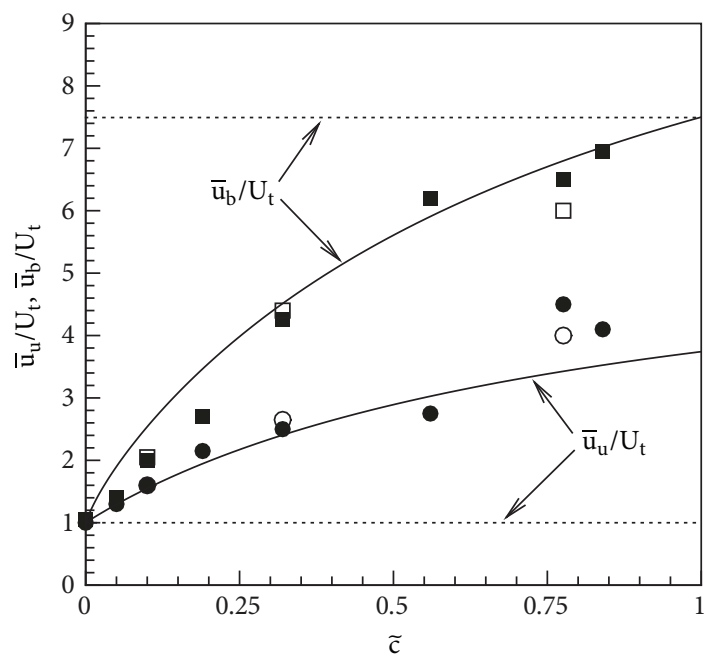

(b)

Figure 3: Comparison of theoretical results for the dimensionless scalar flux and conditional mean velocities (solid curves) with Moss's experimental data [13] (the marks) for a fixed section of a turbulent Bunsen flame.

mean velocities, scalar flux, and stress can be represented schematically by the following expressions:

$$
\begin{aligned}
\bar{u}_{u} & =f_{1}(\widetilde{c}, \Theta), \\
\bar{u}_{b} & =f_{2}(\widetilde{c}, \Theta), \\
\overline{\rho u^{\prime \prime} c^{\prime \prime}} & =f_{3}(\widetilde{c}, \Theta), \\
\overline{\rho u^{\prime \prime} u^{\prime \prime}} & =f_{4}(\widetilde{c}, \Theta) .
\end{aligned}
$$

Here $f_{1}, f_{2}, f_{3}$, and $f_{4}$ are functions that follow directly from the previous equations.

We consider this analysis to be an initial theoretical study of the conditional velocities, mean scalar flux, and stress, performed in the context of the two-fluid approach.

This hydrodynamics analysis is approximate for the following reasons:

(i) We ignore the effects of turbulence within the unburned and burned gases.

(ii) We assume a constant total pressure in the unburned gas.

(iii) We assume that the pressure at each point in the flame is the same.

In the main body of this paper, we consider unclosed differential two-fluid conditional equations and proposed models for the estimation of unknowns that can be used to solve the problem without these assumptions.

A.2. Application of the Two-Fluid Theory to a Bunsen Flame. Although (4)-(7a), (7b), (7c), (7d), and (7e) were formulated for a one-dimensional steady-state flame, they are applicable to a Bunsen flame, since the contribution of the flame brush to the increase in the pressure drop across this turbulent flame is small in comparison with the pressure drop across the flame caused by thermal expansion. Figure 3 is taken from our previous work [15] and shows a comparison of the theoretical results for the dimensionless scalar flux (the left plot) and conditional velocities (the right plot) with Moss's classical experimental data [13], obtained from a fixed section of a Bunsen turbulent flame.

Good agreement between theory and experiment supports the conclusion that hydrodynamic effects dominate in this experiment; i.e., the contribution of the gradient turbulent diffusion can be ignored. The dashed curve in the left-hand plot shows the upper estimate for the scalar flux that gives (7a), using the assumption that the mean conditional velocities across the flame are constant and equal to $\bar{u}_{u}=U_{t}$ and $\bar{u}_{b}=\Theta U_{t}$ (dashed lines in the right-hand plot).

These results show that the countergradient phenomenon and the velocity fluctuations in turbulent flames, which are unusually large for turbulent flows, are hydrodynamic in nature. This means that the scalar flux and stress tensor cannot be presented in gradient form; i.e., the terms "countergradient (negative) turbulent diffusion" and "unusually large turbulent velocity fluctuations" that are sometimes used for premixed flames are misnomers.

\section{B. Criterion for Distinguishing between the Countergradient and Gradient Scalar Flux}

In the general case, the mean conditional velocities $\overline{\vec{u}}_{u}$ and $\overline{\vec{u}}_{b}$ are controlled by hydrodynamics and turbulence, i.e., the type of the scalar flux (gradient, countergradient or neutral), which is governed by the balance between hydrodynamic and turbulent effects. These interactive hydrodynamics and turbulent processes are accurately described in the context of the two-fluid conditionally averaged differential equations. Here, we approximate the scalar flux as the sum of the countergradient hydrodynamic contribution, which we 
describe in the context of the theory considered above, and the contribution of the gradient turbulent diffusion, using the turbulent diffusion coefficient $D_{t}$ :

$$
\begin{aligned}
\overline{\rho u^{\prime \prime} c^{\prime \prime}} & =\left(\overline{\rho u^{\prime \prime} c^{\prime \prime}}\right)_{p-d}+\left(\overline{\rho u^{\prime \prime} c^{\prime \prime}}\right)_{t . d .} \\
& =\rho_{u} U_{t} f_{3}(\bar{c}, \Theta)-\frac{\bar{\rho} D_{t} d \widetilde{c}}{d x} .
\end{aligned}
$$

In order to explain the physical meaning of this assumption, we note that in the case of constant density $\overline{\left(\rho u^{\prime \prime} c^{\prime \prime}\right.}=$ $\rho \overline{u^{\prime} c^{\prime}}$ and $\tilde{c}=\bar{c}$ ) there is no pressure-driven mechanism, and the scalar flux $\rho \overline{u^{\prime} c^{\prime}}$ and difference of the conditional mean velocities $\Delta \bar{u}=\left(\bar{u}_{b}-\bar{u}_{u}\right)$ are controlled by turbulent diffusion $\left(\Delta \bar{u}=\Delta \bar{u}_{t . d .}\right)$ :

$$
\begin{aligned}
\rho \overline{u^{\prime} c^{\prime}} & =\rho \bar{c}(1-\bar{c})\left(\bar{u}_{b}-\bar{u}_{a}\right)=-\frac{\rho D_{t} d \bar{c}}{d x} \Longrightarrow \\
\Delta \bar{u}_{t . d .} & =\frac{-\rho D_{t} d \bar{c} / d x}{[\bar{c}(1-\bar{c})]} .
\end{aligned}
$$

(B.1) means that we represent the difference in the conditional mean velocities in the case of variable density $\Delta \bar{u}$ as a sum of the differences caused by the pressure-driven effect $\Delta \bar{u}_{p-d}$ and turbulent diffusion $\Delta \bar{u}_{t . d}$.

In (B.1), we use elements of the two-fluid conditional averaging and Favre averaging frameworks when representing a scalar flow as the sum of hydrodynamic and turbulent contributions and their estimates. Equation (B.1) is approximate, since it does not include the effect on the flux $\overline{\rho u^{\prime \prime} c^{\prime \prime}}$ of the interaction between hydrodynamic and turbulent processes, which is automatically described by the two-fluid conditionally averaged equations.

B.1. Formulation of the Transition Criterion. Eq. (8) allows us to formulate original local and integral transition criteria:

$$
\begin{aligned}
K_{l o c} & =\frac{\left|\left(\overline{\rho u^{\prime \prime} c^{\prime \prime}}\right)_{p-d}\right|}{\left|\left(\overline{\rho u^{\prime \prime} c^{\prime \prime}}\right)_{t . d .}\right|}=\frac{\left|\rho_{u} U_{t} f_{3}(\bar{c}, \Theta)\right|}{\left|\bar{\rho}(\bar{c}) D_{t} d \widetilde{c} / d x\right|}, \\
K_{\text {int }} & =\frac{\left|\left\langle\left(\overline{\rho u^{\prime \prime} c^{\prime \prime}}\right)_{p-d}\right\rangle\right|}{\left|\left\langle\left(\overline{\rho u^{\prime \prime} c^{\prime \prime}}\right)_{t . d .}\right\rangle\right|} \\
& =\left(\frac{1}{4}\right) \beta\left[\Theta-(2 \Theta-1)^{1 / 2}\right]\left(\frac{U_{t}}{u^{\prime}}\right)\left(\frac{\delta_{t}}{L}\right),
\end{aligned}
$$

where $\left\langle\left(\overline{\rho u^{\prime \prime} c^{\prime \prime}}\right)_{p-d}\right\rangle$ and $\left\langle\left(\overline{\rho u^{\prime \prime} c^{\prime \prime}}\right)_{t . d .}\right\rangle$ are the characteristic values (averaged over the flame section) for pressure-driven hydrodynamic and turbulent diffusion contributions to the scalar flux, and $\beta$ is a constant expected to be of order unity. The local criterion (B.3a), which directly follows from (B.1), shows that the scalar flux $\left(\overline{\rho u^{\prime \prime} c^{\prime \prime}}\right)$ at points inside the turbulent flame for which $K_{l o c}=1, K_{l o c}<1$, and $K_{l o c}>1$ is neutral, gradient, and countergradient, respectively. Analogously, the integral criterion (B.3b) shows that the scalar flux $\left(\overline{\rho u^{\prime \prime} c^{\prime \prime}}\right)$ in the sections of the turbulent flame where $K_{\text {int }}=1, K_{\text {int }}<1$, and $K_{\text {int }}>1$ is neutral, gradient, and countergradient on average, respectively. The local criterion (B.3a) is uniquely defined in the context of the simple theory considered above and its representation (B.1). At the same time, different estimates of the characteristic values of hydrodynamic and turbulent contributions may appear in (B.3b).

The assumptions that we used to obtain the integral criterion (B.3b) are as follows:

(i) We assumed that the characteristic value of the mean flux $\left\langle\left(\overline{\rho u^{\prime \prime} c^{\prime \prime}}\right)_{p-d}\right\rangle$ corresponds to the flux described by (A.4a) with $\widetilde{c}^{\circ}=0.5, \widetilde{c}^{\circ}\left(1-\widetilde{c}^{\circ}\right)=1 / 4$, and $\bar{\rho}=$ $\bar{\rho}\left(\widetilde{c}^{\circ}\right)$, although for simplification, we estimated the conditional velocities $\bar{u}_{u}$ and $\bar{u}_{b}$ using dimensionless equations (A.2a) and (A.2b) with $\bar{c}=1$ or $\tilde{c}=1$. This results in the dimensionless velocities $\bar{u}_{b}=\Theta$ and $\bar{u}_{u}=\left[\Theta-(2 \Theta-1)^{1 / 2}\right]$, which somewhat overestimate the difference in velocities described by (A.2a) and (A.2b) (see Figure 3(b)).

(ii) The estimation of the dimension diffusion flux is $\left\langle\bar{\rho} D_{t} d \widetilde{c} / d x\right\rangle \approx \bar{\rho}\left(c^{\circ}\right) u^{\prime} L\left(1 / \delta_{t}\right)$.

Comparing our and analogous criterion of transition $N_{B}=\left(\tau S_{L}\right) /\left(2 \alpha u^{\prime}\right)=1$ (where $\tau=(\Theta-1)$ and $\alpha$ (in our case, $\left.\delta_{L} / L \longrightarrow 0\right)$ is a constant expected to be of order unity) as proposed in [24], we notice that the latter was not based on a comparison of the pressure-driven hydrodynamic and turbulent contributions to the scalar flux. Hence, the criterion $N_{B}$ contains the parameters of the turbulent flame $U_{t}$ and $\delta_{t}$, which affect the hydrodynamics and turbulent effects, respectively; it therefore cannot predict, for example, the observed transition from the gradient scalar flux to the countergradient flux along the Bunsen flame, where $u^{\prime}$ is nearly constant.

B.2. Qualitative Application of the Criterion to OneDimensional and Bunsen Flames. We first apply these criteria to the one-dimensional steady-state flame where $\Theta>>1$. The speed of the premixed flame with strong turbulence $u^{\prime}>S_{L}$ does not depend on $S_{L}$ and is equal to $U_{t} \sim u^{\prime}$ in accordance with the Damköhler qualitative estimation [25], which was confirmed and clarified theoretically $\left(U_{t} \cong u^{\prime}\right)$ in [22]. For the case of a typical stoichiometric mixture with $\Theta=7\left(T_{u}=300 \mathrm{~K}\right.$ and $\left.T_{b}=2100 \mathrm{~K}\right)$, the integral criterion $K_{\text {int }}$ (B.3b) shows that a transition takes place (assuming in (B.3b) that $\beta=1$ ) in the section where $\delta_{t} / L \approx 1$. We believe that the width of this flame should be $\delta_{t} / L \sim(5-10)$; i.e., the scalar flux is integrally countergradient. At the same time, in accordance with the local criterion (B.3a), the scalar flux may be gradient in the vicinity of the front edge of the flame, since the difference in the velocities $\bar{u}_{u}$ and $\bar{u}_{b}$ is small in this vicinity, in accordance with the theory (see Figure 3(b)).

The turbulent Bunsen flame is characterized by an increasing width and at the same time a constant angle inclination to the mean flow, i.e., nearly constant speed $U_{t}$ along the flame. This means that the gradient turbulent component $\left|\left\langle\left(\overline{\rho u^{\prime \prime} c^{\prime \prime}}\right)_{t . d .}\right\rangle\right|$ in the integral criterion (B.3b) is reduced 


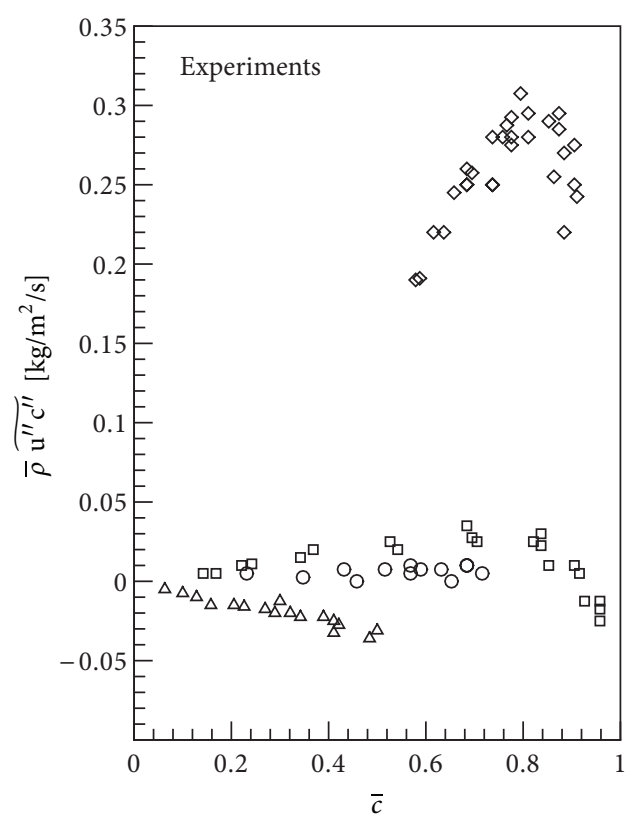

(a)

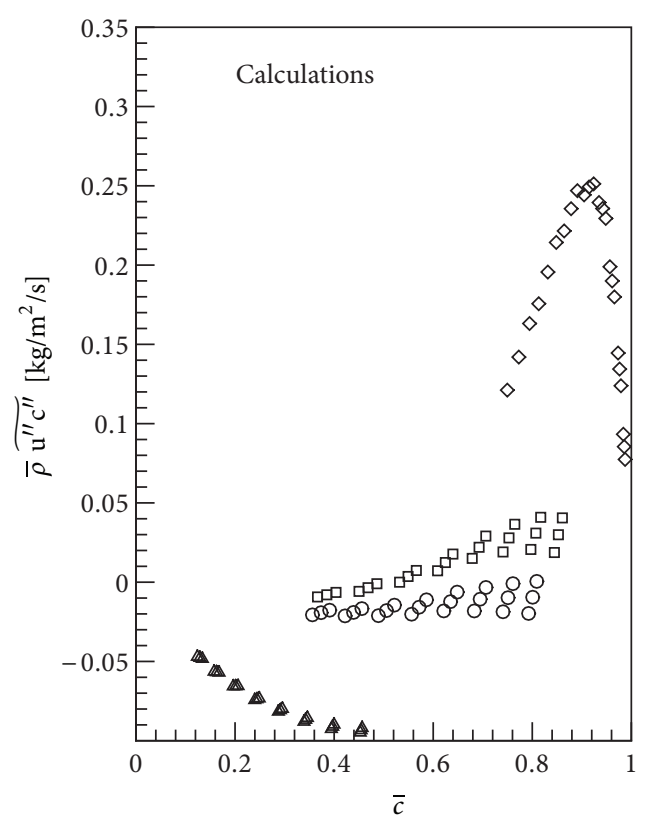

(b)

FIGURE 4: Comparison of experimental [14] (LHS) and theoretically estimated [15] (RHS) scalar flux in the fixed section of the turbulent Bunsen flame.

along the direction of the flame, while the hydrodynamic component $\left|\left\langle\left(\overline{\rho u^{\prime \prime} c^{\prime \prime}}\right)_{p-d}\right\rangle\right|$ remains nearly constant. In the case of a sufficiently large countergradient $\left|\left\langle\left(\overline{\rho u^{\prime \prime} c^{\prime \prime}}\right)_{p-d}\right\rangle\right|$ (intensive combustion $U_{t} \sim u^{\prime}$ and a large ratio of the densities $\rho_{u} / \rho_{b} \gg 1$ ), there will be a transition from gradient to countergradient scalar flux.

In [22], we obtained the formula $U_{t} \approx\left(u^{\prime} S_{L}\right)^{1 / 2}$ for the speed of the transient flame under conditions of strong turbulence $u^{\prime}>S_{L}$ with increasing width and constant speed; i.e., we must substitute $U_{t} / u^{\prime} \sim\left(S_{L} / u^{\prime}\right)^{1 / 2}$ in (B.3b). The theoretical concept of this transient flame is based on the assumption of statistical equilibrium of small-scale wrinkles and at the same time disequilibrium of large-scale wrinkles in the instantaneous turbulent flame at the intermediate asymptotic stage. We note that the theory for a one-dimensional steady-state flame is applicable to the Bunsen flame, since the effect of the increase in flame width on the pressure drop across the flame is small. In a typical case with $\Theta=7$ and $u^{\prime} / S_{L}=5$, the transition takes place in the section where $\delta_{t} / L \approx 3$; i.e., the transition can take place because the flame width can reach substantially larger values.

\section{Gradient and Countergradient Scalar Flux in Bunsen and Impinging Flames}

Previous experimental studies of the scalar flux in the Bunsen flame usually report the results of cross-section measurements, which are used in Figure 3, for example, where the scalar flux measured in [13] was countergradient. In [14], Bilger et al. studied the Bunsen flame, where the scalar flux in a fixed cross-section was countergradient, neutral, or gradient depending on the turbulent parameters of the flow and the mixture composition. In [15], we compared these experimental data with our theoretical analysis of the scalar flux in the Bunsen flame. We briefly present this comparison for the reader's convenience and also consider the results of a study of the scalar flux in free-standing impinging flames and those close to the wall. In both cases, we use the representation (B.1), in which the estimation of the hydrodynamic countergradient contribution is based on the two-fluid one-dimensional theory.

We hope that these data provide an additional argument in favor of the two-fluid conditional averaging framework developed in later sections.

C.1. Scalar Flux Regimes in the Bunsen Flame. In Figure 4 taken from our paper [15] the left plot represent in the coordinates $\overline{\rho u^{\prime \prime} c^{\prime \prime}}=f(\bar{c})$ the experimental data for the scalar flux in the fixed cross-section of the Bunsen flame obtained in [14], and in the right plot the results of our estimations. The velocity fluctuations $u^{\prime}$ and ratios $u^{\prime} / S_{L}$ corresponding to different marks in the Figure 4 are as follows: $u^{\prime}=0.85 \mathrm{~m} / \mathrm{s}$ and $u^{\prime} / S_{L}=3.6$ (rhombs); $u^{\prime}=$ $0.53 \mathrm{~m} / \mathrm{s}$ and $u^{\prime} / S_{L}=3.1$ (squares); $u^{\prime}=0.83 \mathrm{~m} / \mathrm{s}$ and $u^{\prime} / S_{L}=$ 4.9 (circles); $u^{\prime}=0.79 \mathrm{~m} / \mathrm{s}$ and $u^{\prime} / S_{L}=8.8$ (triangles).

In regimes with similar values of the velocity fluctuations in the flow $\left(u^{\prime}=0.79 \mathrm{~m} / \mathrm{s}, u^{\prime}=0.83 \mathrm{~m} / \mathrm{s}\right.$, and $\left.u^{\prime}=0.85 \mathrm{~m} / \mathrm{s}\right)$, the contributions of the gradient turbulent diffusion to the scalar flux were similar to each other, while the contributions of the countergradient hydrodynamic effect were different. The latter increased with the values of the laminar flame speed with variations in the mixture composition as follows: $S_{L}=0.09 \mathrm{~m} / \mathrm{s}\left(u^{\prime}=0.79 \mathrm{~m} / \mathrm{s}, u^{\prime} / S_{L}=8.8\right) ; S_{L}=0.17 \mathrm{~m} / \mathrm{s}$ 

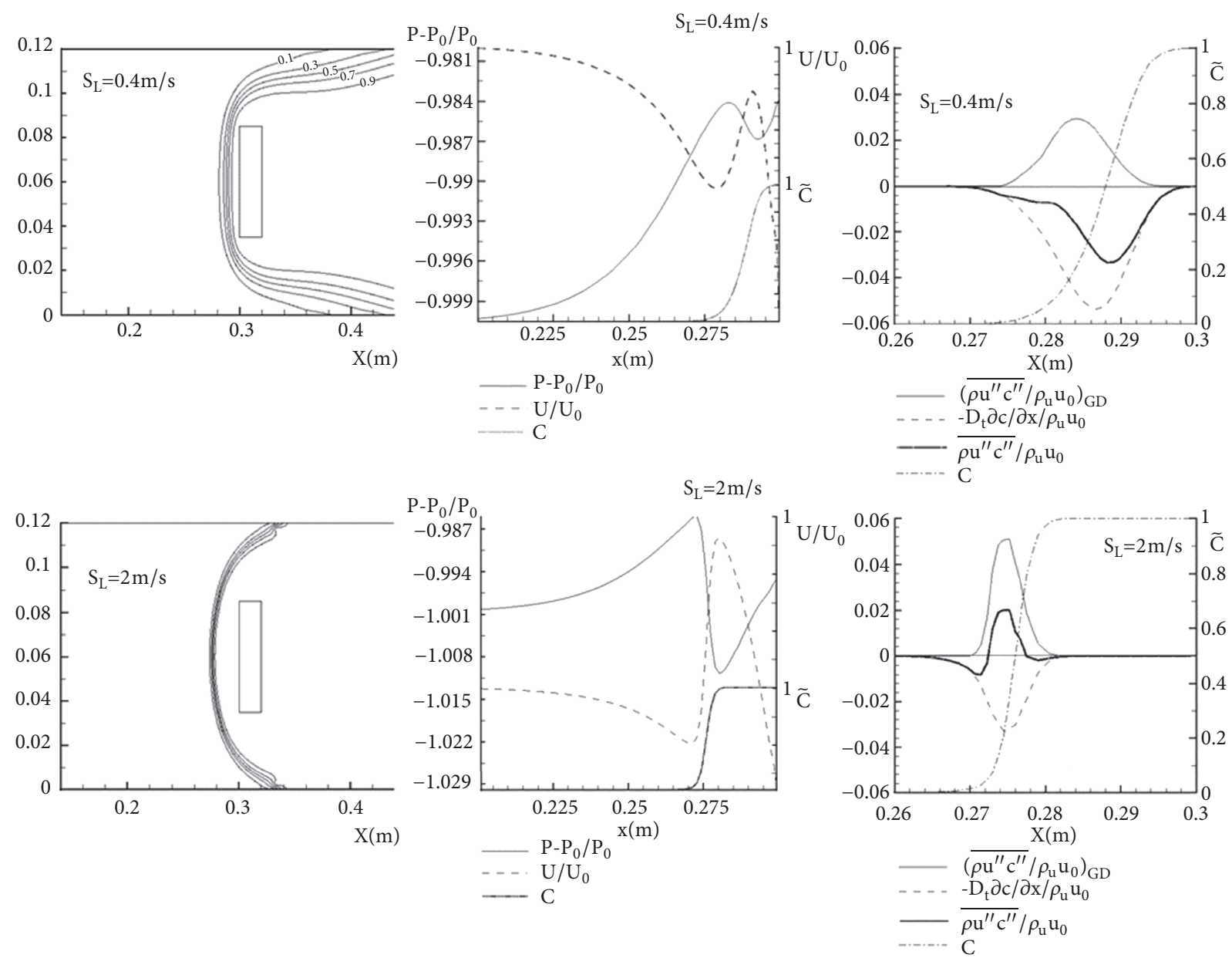

Figure 5: Configurations and profiles of the parameters of the flame close to the wall (upper plots) and the free-standing flame (lower plots).

$\left(u^{\prime}=0.83 \mathrm{~m} / \mathrm{s}, u^{\prime} / S_{L}=4.9\right) ;$ and $S_{L}=0.24 \mathrm{~m} / \mathrm{s}\left(u^{\prime}=\right.$ $\left.0.85 \mathrm{~m} / \mathrm{s}, u^{\prime} / S_{L}=3.6\right)$. The hydrodynamic effect is smaller for the mixture with a smaller laminar flame speed $S_{L}$, due to the lower turbulent speed $U_{t}$ and ratio $\Theta=\rho_{u} / \rho_{b}$, and hence the smaller pressure drops across the turbulent flame $\Delta p=\rho_{u} U_{t}^{2}(\Theta-1)$, and vice versa. When $S_{L}=0.09 \mathrm{~m} / \mathrm{s}$, the turbulent diffusion component dominated, and the scalar flux had a gradient direction; when $S_{L}=0.24 \mathrm{~m} / \mathrm{s}$, the hydrodynamic component dominated, and the scalar flux had a countergradient direction. For an intermediate value of the speed, $S_{L}=0.17 \mathrm{~m} / \mathrm{s}$, the contributions of the hydrodynamics and turbulence effects were comparable, resulting in nearneutral scalar flux. In the fourth regime shown in Figure 4, the velocity fluctuations were smaller $\left(u^{\prime}=0.53 \mathrm{~m} / \mathrm{s}\right)$, but the laminar flame speed had the same intermediate value $S_{L}=0.17 \mathrm{~m} / \mathrm{s}\left(u^{\prime} / S_{L}=3.1\right)$. Hence, both contributions were smaller (the turbulent effect was smaller due to a smaller value of $u^{\prime}$, while the hydrodynamic effect was smaller due to a smaller value of $U_{t}$, caused by smaller value of the speed $u^{\prime}$ ), resulting in the near-neutral scalar flux in the experiments and calculations shown in Figure 4.
C.2. Scalar Flux in Close-to-Wall and Free-Standing Impinging Flames. Figure 5 (a reproduction of Figure 31 in [19]) illustrates the potential of the two-fluid approach in the theoretically grounded modeling of the scalar flux in the impinging flame. This figure shows the results of numerical simulations of the two 2D flames in the channel (close to the obstacle and free-standing impinging flames). The simulations were performed by Valirio Battaglia using the commercial code "Fluent" based on the TFC combustion model [17] (see [26] for more details).

The flow and turbulence parameters in both cases were the same $\left(U=30 \mathrm{~m} / \mathrm{s}, u^{\prime}=8 \mathrm{~m} / \mathrm{s}, L=0.54 \mathrm{~cm}, p=\right.$ 1atm, $\left.T=300 \mathrm{~K}, \rho_{u} / \rho_{b}=7\right)$, although the combustible mixtures were different. Close-to-obstacle and free-standing flames had laminar flame speeds $S_{L}=0.4 \mathrm{~cm} / \mathrm{s}$ and $2 \mathrm{~cm} / \mathrm{s}$, respectively.

The left-hand images show the geometry of the channel and obstacle (a 2D plate), and the isosurfaces $\widetilde{c}=$ const, which show the configuration of the flames. The central pictures show the dimensionless mean pressure $\left(\bar{p}-p_{0}\right) / \bar{p}$ (solid line), mean velocity $U / U_{0}$ (dotted line), and the progress variable 
$\widetilde{c}$ (dashed-dotted line) along the central axis. The left-hand image shows the dimensionless scalar fluxes, which in our notation are the countergradient pressure-driven contribution $\left(\overline{\rho u^{\prime \prime} c^{\prime \prime}}\right)_{p-d} / \rho_{u} U_{0}$ (solid line), the gradient turbulent diffusion contribution $\left(\overline{\rho u^{\prime \prime} c^{\prime \prime}}\right)_{t . d .} / \rho_{u} U_{0}$ or $\left(-\rho_{u} D_{t} \partial \widetilde{c} / \partial x\right) / \rho_{u} U_{0}$ (dotted line), and the summary scalar flux $\left(\overline{\rho u^{\prime \prime} c^{\prime \prime}}\right) / \rho_{u} U_{0}$ (bold solid line). It can be seen that the direction of the scalar flux in the flame close to the obstacle corresponds to gradient diffusion, while the scalar flux in the free-standing flame is countergradient except for the zone adjacent to the leading edge of the flame. In [26], we discussed the reason for the domination of turbulent diffusion in the head of the flame.

From a theoretical viewpoint, these results are reasonable since they correlate with the profile of the pressure, in contrast to the results for the close-to-the-wall and free-standing impinging flames mentioned in Section 3.1 and obtained in [4] in the context of the Favre averaging framework.

Our approximate approach using (9a) and (9b) for the estimation of the scalar flux in the impinging flame was validated in [27] against known experimental data for two impinging flames situated at different distances from the wall [28]. In both flames, the experimental and calculated scalar fluxes were countergradient, but in the flame that was closer to the wall, the level of the scalar flux was lower due to the smaller pressure drop against the flame (Figure 3 in [27]).

\section{Summary of Appendix}

The results of the preliminary investigation of the two-fluid approach considered in this appendix were based on an approximate representation of the scalar flow in the form of a sum of hydrodynamic and turbulent contributions and can help us to better understand the potentialities of the two-fluid approach and the problems arising from this, which facilitates a deeper study of this issue. Taking into account the basic advantages of the two-fluid approach demonstrated above and bearing in mind the well-documented fact that, in many practical cases, instantaneous combustion occurs in highly curved, thin layers, we believe that the two-fluid conditional averaging framework shows significant promise for modeling applications. In particular, it seems reasonable to reformulate the TFC combustion model, which is represented in the commercial packages Fluent and CFX, in the context of the two-fluid conditional averaging paradigm.

\section{Nomenclature}

$\begin{array}{ll}\text { BML: } & \text { Bray-Moss-Libby } \\ c: & \text { Progress variable } \\ D a=\tau_{t} / \tau_{c h}: & \text { Damköhler number } \\ K a=\left(\delta_{L} / \eta\right)^{2}: & \text { Karlovitz number } \\ \widetilde{k}, \bar{k}_{u}, \bar{k}_{b}: & \text { Favre- and conditionally averaged } \\ & \text { turbulent kinetic energy } \\ L: & \text { Integral scale of turbulence } \\ \vec{n}: & \text { Unit normal vector to instantaneous } \\ & \text { flame } \\ P(c): & \text { PDF of progress variable } \\ \text { PDF: } & \text { Probability density function }\end{array}$

$P_{u}, P_{b}: \quad$ Probabilities of unburned and

burned gases

$\bar{p}_{u}, \bar{p}_{b}: \quad$ Conditional mean pressures in unburned and burned gases

$\operatorname{Re}_{t}=u^{\prime} L / v$ : Turbulent Reynolds number

$S_{L}$ : $\quad$ Speed of laminar flame

$u^{\prime}=\left(\overline{u^{\prime 2}}\right)^{1 / 2}$ : Root-mean-square velocity

fluctuation

$\overline{\vec{u}}_{s u}, \bar{u}_{s b}: \quad$ Surface-averaged speeds at the edges

$\overline{\vec{u}}_{u}, \bar{u}_{b}: \quad$ of an instantaneous flame

unburned and burned gases

$U_{f}$ : Thickened flamelet propagation

$U_{t}: \quad$ Turbulent premixed flame propagation speed.

\section{Greek Symbols}

\begin{tabular}{|c|c|}
\hline$\delta_{f}:$ & Width of thickened flamelet \\
\hline$\delta_{L}:$ & Width of laminar flame \\
\hline$\delta_{t}:$ & Width of turbulent flame \\
\hline$\widetilde{\mathcal{\varepsilon}} ; \bar{\varepsilon}_{u}, \bar{\varepsilon}_{b}:$ & $\begin{array}{l}\text { Favre- and conditionally averaged } \\
\text { dissipation rates }\end{array}$ \\
\hline$\eta=L \operatorname{Re}_{t}^{-3 / 4}:$ & Kolmogorov microscale \\
\hline$\Theta=\rho_{u} / \rho_{b}$ & $\begin{array}{l}\text { Ratio of densities of unburned and } \\
\text { burned gases }\end{array}$ \\
\hline$v:$ & Kinematic viscosity coefficient \\
\hline$\rho:$ & Density \\
\hline$\rho_{u}, \rho_{b}:$ & $\begin{array}{l}\text { Density of unburned and burned } \\
\text { gases }\end{array}$ \\
\hline$\rho \vec{u}^{\prime \prime} c^{\prime \prime}:$ & Mean scalar flux \\
\hline$\rho \vec{u}^{\prime \prime} \vec{u}^{\prime \prime}:$ & Mean stress tensor \\
\hline$\overline{\rho W}:$ & Mean chemical source \\
\hline$\Sigma_{f}:$ & Flame surface density \\
\hline$\tau_{c h}=\chi / S_{L}^{2}$ & Chemical combustion time \\
\hline$\tau_{i j, u}, \tau_{i j, b}$ & Conditional Reynolds stresses \\
\hline$\tau_{t}=L / u^{\prime}:$ & $\begin{array}{l}\text { Turbulent time } \\
\text { Molecular heat transfer coeffic }\end{array}$ \\
\hline
\end{tabular}

\section{Data Availability}

No data were used to support this study.

\section{Conflicts of Interest}

The author declares no conflicts of interest.

\section{Acknowledgments}

I have benefited greatly from the discussions about this topic with Ken Bray (who encouraged me in due time to develop this alternative approach), Denis Veynante (who looked through a preliminary version of the manuscript), and my former colleague Fernando Biagioli. 


\section{References}

[1] R. W. Bilger, S. B. Pope, K. N. C. Bray, and J. F. Driscoll, "Paradigms in turbulent combustion research," Proceedings of the Combustion Institute, vol. 30, no. 1, pp. 21-42, 2005.

[2] K. N. C. Bray, P. A. Libby, and J. B. Moss, "Unified modeling approach for premixed turbulent combustion-part i: general formulation," Combustion and Flame, vol. 61, no. 1, pp. 87-102, 1985.

[3] S. B. Pope, "Turbulent premixed flames," Annual Review of Fluid Mechanics, vol. 19, no. 1, pp. 237-270, 1987.

[4] K. N. C. Bray, M. Champion, and P. A. Libby, "Premixed flames in stagnating turbulence part IV: a new theory for the Reynolds stresses and Reynolds fluxes applied to impinging flows," Combustion and Flame, vol. 120, no. 1-2, pp. 1-18, 2000.

[5] F. A. Williams, "The role of theory in combustion science," Symposium (International) on Combustion, vol. 24, no. 1, pp. 117, 1992.

[6] D. B. Spalding, "The two-fluid model of turbulence applied to combustion phenomena," AIAA Journal, vol. 24, no. 6, pp. 876$884,1986$.

[7] H. G. Weller, "The developing of a new flame area combustion model using conditional averaging," Thermo-Fluids Section Report TF/9307, Department of Mechanical Engineering, Imperial College of Science Technology and Medicine, 1993, http://citeseerx.ist.psu.edu/viewdoc/download?doi=10.1.1.45 $.6303 \&$ amp;rep $=$ rep1\&amp;type $=$ pdf.

[8] E. Lee and K. Y. Huh, "Zone conditional modeling of premixed turbulent flames at a high Damköhler number," Combustion and Flame, vol. 138, no. 3, pp. 211-224, 2004.

[9] V. L. Zimont, "A splitting method and alternative unclosed twofluid conditional equations of turbulent premixed combustion without surface-averaged unknowns," Combustion and Flame, vol. 192, pp. 221-223, 2018.

[10] J. F. Driscoll, “Turbulent premixed combustion: flamelet structure and its effect on turbulent burning velocities," Progress in Energy and Combustion Science, vol. 34, no. 1, pp. 91-134, 2008.

[11] F. Dinkelacker, A. Soika, D. Most et al., "Structure of locally quenched highly turbulent lean premixed flames," Symposium (International) on Combustion, vol. 27, no. 1, pp. 857-865, 1998.

[12] V. Zimont, "Unclosed Favre-averaged equation for the chemical source and an analytical formulation of the problem of turbulent premixed combustion in the flamelet regime," Combustion and Flame, vol. 162, no. 3, pp. 874-875, 2015.

[13] J. B. Moss, "Simultaneous measurements of concentration and velocity in an open premixed turbulent flame," Combustion Science and Technology, vol. 22, no. 3-4, pp. 119-129, 1980.

[14] J. H. Frank, P. A. Kalt, and R. W. Bilger, "Measurements of conditional velocities in turbulent premixed flames by simultaneous OH PLIF and PIV," Combustion and Flame, vol. 116, no. 1-2, pp. 220-232, 1999.

[15] V. L. Zimont and F. Biagioli, "Gradient, counter-gradient transport and their transition in turbulent premixed flames," Combustion Theory and Modelling, vol. 6, no. 1, pp. 79-101, 2002.

[16] V. L. Zimont, "Theory of turbulent combustion of a homogeneous fuel mixture at high reynolds numbers," Combustion, Explosion and Shock Waves, vol. 15, no. 3, pp. 305-311, 1979.

[17] V. Zimont, "Gas premixed combustion at high turbulence. Turbulent flame closure combustion model," Experimental Thermal and Fluid Science, vol. 21, no. 1-3, pp. 179-186, 2000.
[18] A. W. Skiba, T. M. Wabel, C. D. Carter, S. D. Hammack, J. E. Temme, and J. F. Driscoll, "Premixed flames subjected to extreme levels of turbulence part I: Flame structure and a new measured regime diagram," Combustion and Flame, vol. 189, pp. 407-432, 2018.

[19] V. L. Zimont, "Kolmogorovs legacy and turbulent premixed combustion modelling," in New Developments in Combustion Research, W. J. Carey, Ed., p. 93, Nova Science Publishers, Inc, New York, NY, USA, 2006.

[20] E. Lee, Y. H. Im, and K. Y. Huh, "Zone conditional analysis of a freely propagating one-dimensional turbulent premixed flame," Proceedings of the Combustion Institute, vol. 30, no. 1, pp. 851857, 2005.

[21] D. C. Wilcox, Turbulence Modeling for CFD, DCW Industries Inc., Calif, USA, 3rd edition, 2006.

[22] V. L. Zimont, "Damkohler-Shelkin paradox in the theory of turbulent flame propagation, and a concept of the premixed flame at the intermediate asymptotic stage," Flow, Turbulence and Combustion, vol. 97, no. 3, pp. 875-912, 2016.

[23] A. N. Lipatnikov, "Conditionally averaged balance equations for modeling premixed turbulent combustion in flamelet regime," Combustion and Flame, vol. 152, no. 4, pp. 529-547, 2008.

[24] D. Veynante, A. Trouvé, K. N. Bray, and T. Mantel, "Gradient and counter-gradient scalar transport in turbulent premixed flames," Journal of Fluid Mechanics, vol. 332, pp. 263-293, 1997.

[25] G. Damköhler, "Der einflußder turbulenz auf die flammengeschwindigkeit in gasgemischen," Zeitschrift Fur Elektrochemie und Angewandte Physikalische Chemie, vol. 46, no. 11, pp. 601-626, 1940.

[26] V. L. Zimont and V. Battaglia, "Joint RANS/LES approach to premixed flame modelling in the context of the tfc combustion model," Flow, Turbulence and Combustion, vol. 77, no. 1-4, pp. 305-331, 2006.

[27] F. Biagioli and V. L. Zimont, "Gasdynamics modeling of countergradient transport in open and impinging turbulent premixed flames," Proceedings of the Combustion Institute, vol. 29, no. 2, pp. 2087-2095, 2002.

[28] S. C. Li, P. A. Libby, and F. Williams, "Experimental investigation of a premixed flame in an impinging turbulent stream," Symposium (International) on Combustion, vol. 25, no. 1, pp. 1207-1214, 1994. 


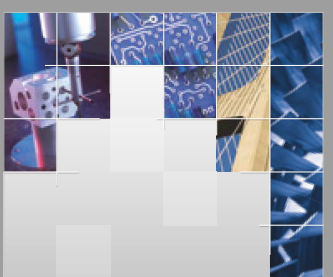

\section{Enfincering}
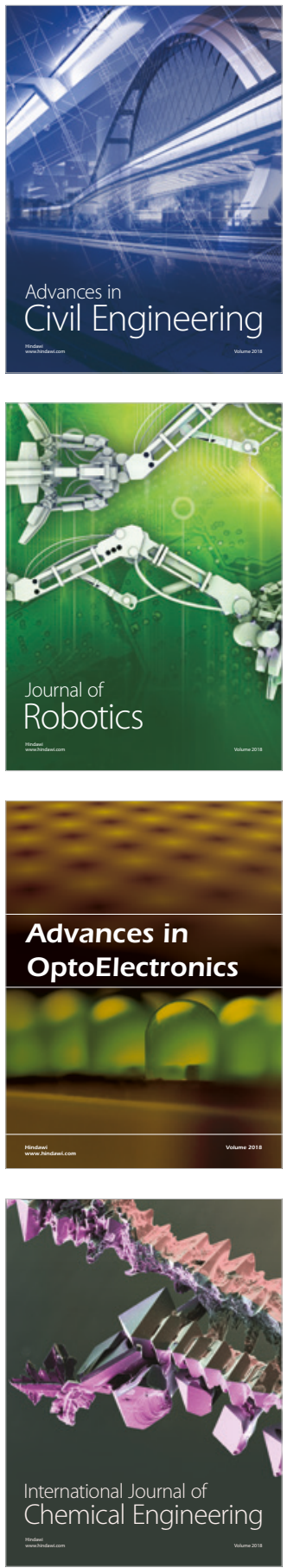

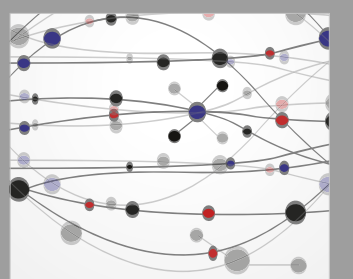

\section{Rotating \\ Machinery}

The Scientific World Journal

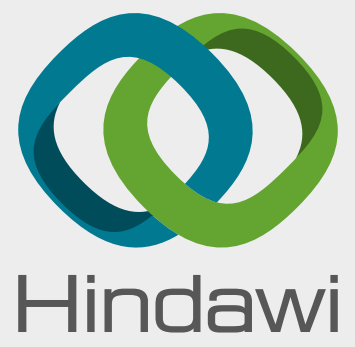

Submit your manuscripts at

www.hindawi.com
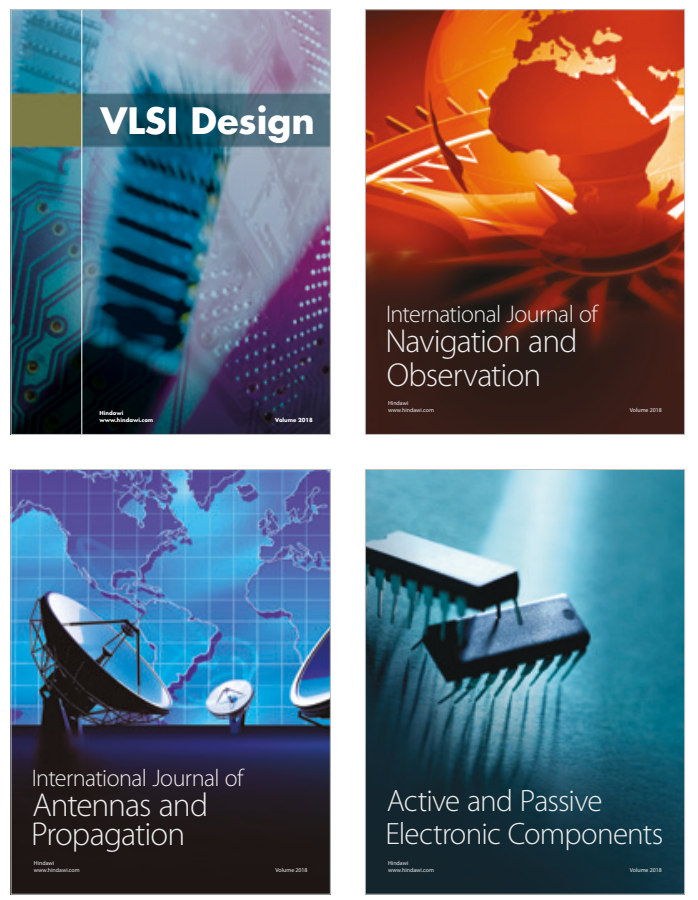
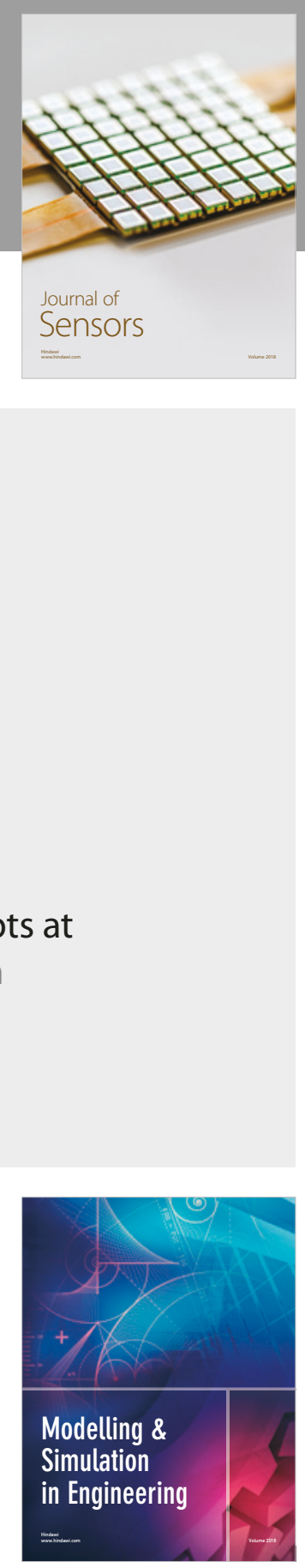

\section{Advances \\ Multimedia}
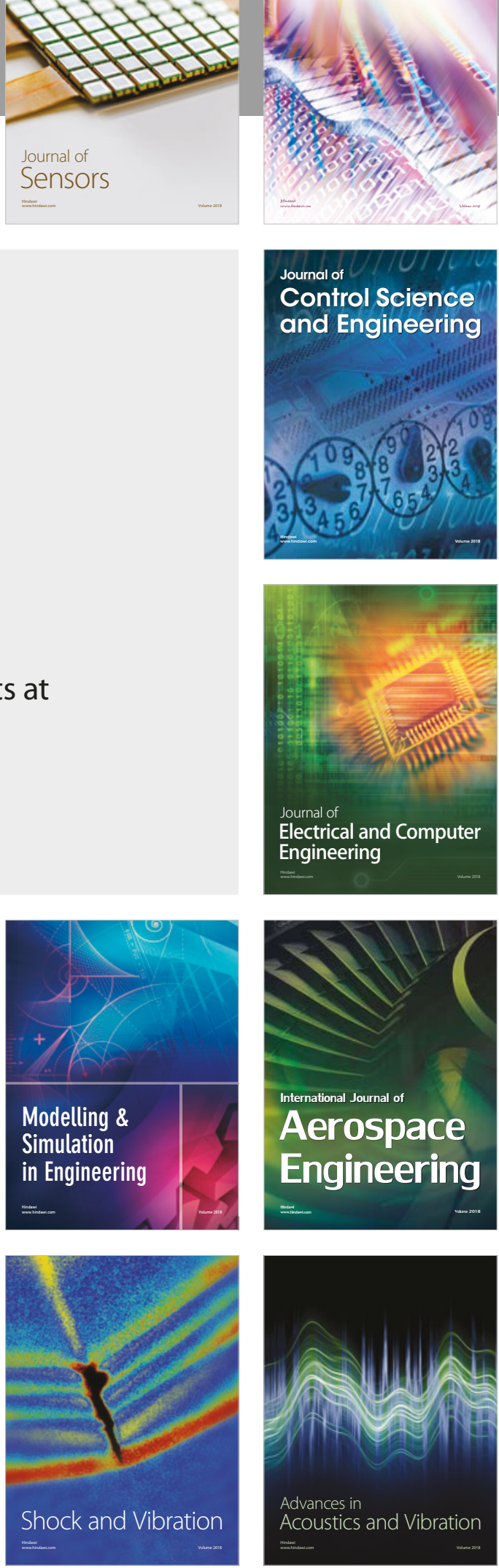